Homology, Homotopy and Applications, vol.21(1), 2019, pp.187-212

\title{
DELIGNE-BEILINSON CYCLE MAPS FOR LICHTENBAUM COHOMOLOGY
}

\author{
TOHRU KOHRITA
}

\author{
(communicated by Charles A. Weibel)
}

\begin{abstract}
We define Deligne-Beilinson cycle maps for Lichtenbaum cohomology of arbitrary complex algebraic varieties and show that the analogues of the Abel-Jacobi theorem and the Lefschetz theorem on $(1,1)$-cycles hold for any complex algebraic variety if we replace the divisor class group with Voevodsky's motivic cohomology with compact supports. For more general indices, we study the torsion part of the cycle maps. We also characterize the algebraic part of Griffiths's intermediate Jacobians by a universal property.
\end{abstract}

\section{Introduction}

Suppose $X$ is a smooth projective complex algebraic variety. The Chow group $C H^{r}(X)$ of cycles of codimension $r$ admits a cycle map $c l^{\mathcal{D B}}$ to the Deligne-Beilinson cohomology $H_{\mathcal{D} \mathcal{B}}^{2 r}(X, \mathbb{Z}(r))$ which is compatible with the Abel-Jacobi map $A J$ and the Betti cycle map $c l^{B}[\mathbf{E V 8 8}$, Section 7]:

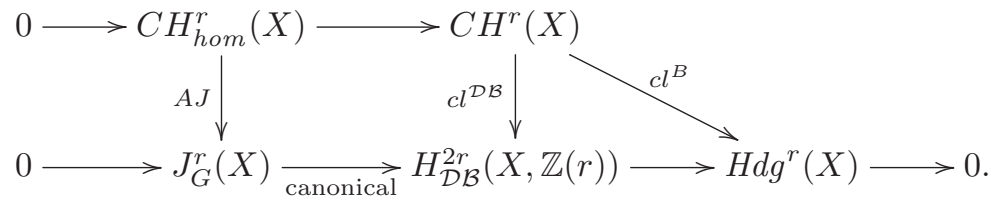

Here, the homological part $C H_{h o m}^{r}(X)$ is by definition the kernel of $c l^{B}$, the group (in fact, a complex torus) $J_{G}^{r}(X)$ is the $r$-th Griffiths intermediate Jacobian of $X$ and $H d g^{r}(X)$ is the group of Hodge $r$-cycles.

The purpose of this article is to extend the diagram (1) to Lichtenbaum cohomology of arbitrary complex varieties $X$, and study the Abel-Jacobi map and Betti cycle map through the Deligne-Beilinson cycle map in the étale context.

The diagram (1) indicates, for example, that the Deligne-Beilinson cycle map $c l^{\mathcal{D B}}$ is an isomorphism if and only if $A J$ is an isomorphism and the integral Hodge conjecture is true, i.e. the image of $c l^{B}$ is the whole of $H d g^{r}(X)$. These are not true in general [Mum68, AH62], but they are for $r=1$. In fact, our Theorem 1.1 is obtained via this equivalence. Although the Hodge conjecture with integral coefficients fails, we would like to recall that, by replacing Chow groups with Lichtenbaum cohomology,

Received October 5, 2017, revised March 5, 2018, June 20, 2018; published on October 10, 2018. 2010 Mathematics Subject Classification: 14F42, 14C30, $19 \mathrm{E} 15$.

Key words and phrases: étale motivic cohomology, Deligne cohomology, cycle map.

Article available at http://dx.doi.org/10.4310/HHA.2019.v21.n1.a9

Copyright (C) 2018, Tohru Kohrita. Permission to copy for private use granted. 
[RS16] formulated an integral version of the Hodge conjecture and showed that their formulation is equivalent to the classical Hodge conjecture with rational coefficients. This may suggest the usefulness of Lichtenbaum cohomology.

With Bloch's construction of cycle maps for his higher Chow groups [Blo86], the diagram (1) naturally extends to higher Chow groups of smooth projective complex varieties. In this context, the Abel-Jacobi maps are defined as restrictions of the Deligne-Beilinson cycle maps. With Rosenschon and Srinivas's cycle maps for Lichtenbaum cohomology defined in [RS16], the diagram (1) also has an analogue for Lichtenbaum cohomology of smooth projective complex varieties. Moreover, as shown in there, Bloch's cycle maps for higher Chow groups factor through RosenschonSrinivas's cycle maps for Lichtenbaum cohomology.

Our generalization of the diagram (1) is formulated with eh hypercohomology of [Gei06]. In this article, by Lichtenbaum cohomology $H_{L}^{m}(X, \mathbb{Z}(n))$ (resp., with compact supports $H_{c, L}^{m}(X, \mathbb{Z}(n))$ ), we mean the eh hypercohomology (resp., with compact supports) of the eh sheafification of the Suslin-Friedlander motivic complex $\mathbb{Z}(n)^{S F}$ (see [MVW06, Lecture 16]). The Deligne-Beilinson cohomology $H_{\mathcal{D B}}^{m}(X, \mathbb{Z}(n)$ ) (resp., with compact supports $H_{c, \mathcal{D} B}^{m}(X, \mathbb{Z}(n))$ ) means eh hypercohomology (resp., with compact supports) of the eh sheafification of a certain complex of Zariski sheaves defined in [Bei84, (1.6.5)] (see Definition 3.5).

For an arbitrary complex algebraic variety $X$, we construct the following analogue of the diagram (1), and also the version without compact supports (see the diagram (17)):

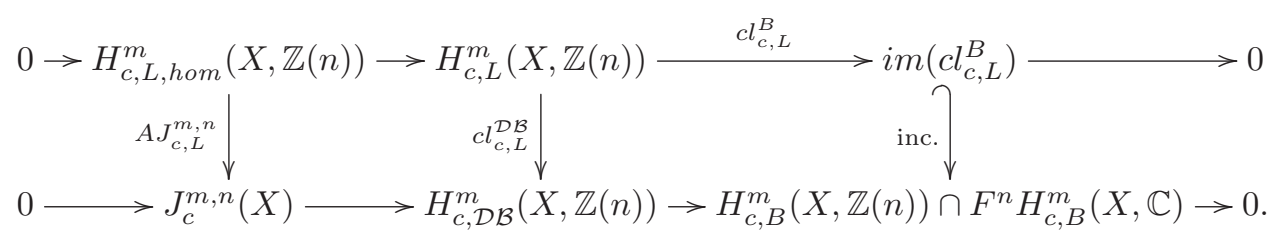

Here, $F^{n} H_{c, B}^{m}(X, \mathbb{C})$ is the $n$-th Hodge filtration. $c l_{c, L}^{\mathcal{D B}}: H_{c, L}^{m}(X, \mathbb{Z}(n)) \rightarrow H_{c, \mathcal{D} \mathcal{B}}^{m}$ $(X, \mathbb{Z}(n))$ is the Deligne-Beilinson cycle map (with compact support) defined in Definition 5.3. The group $H_{c, L, h o m}^{m}(X, \mathbb{Z}(n))$ is the kernel of the Betti cycle map $c l_{c, L}^{B}: H_{c, L}^{m}(X, \mathbb{Z}(n)) \rightarrow H_{c, B}^{m}(X, \mathbb{Z}(n))$ whose definition is analogous to that of $c l_{c, L}^{\mathcal{D B}}$. The map $A J_{c, L}^{m, n}$ is defined as the restriction of $c l_{c, L}^{\mathcal{D B}}$, and its target $J_{c}^{m, n}(X)$ is the Carlson $n$-th intermediate Jacobian associated with the mixed Hodge structure of $H_{c, B}^{m-1}(X, \mathbb{Z}(n))$ [Car79]. The homological part $H_{c, L, h o m}^{m}(X, \mathbb{Z}(n))$ agrees with the subgroup of all divisible elements of $H_{c, L}^{m}(X, \mathbb{Z}(n))$ (Remark 6.2).

The cycle maps $c l_{c, L}^{\mathcal{D B}}$ and $c l_{c, L}^{B}$ are constructed in the derived category of sheaves on the eh site $(S c h / \mathbb{C})_{e h}$. If $X$ is smooth and proper, our cycle maps agree with the usual cycle maps for Chow groups after the composition with the canonical map $C H^{r}(X) \rightarrow H_{L}^{2 r}(X, \mathbb{Z}(r))$. This follows from the construction of our cycle maps in Section 5 (Theorem 5.2).

With the above diagram for $(m, n)=(2,1)$, we can generalize the Abel-Jacobi theorem and the Lefschetz theorem on $(1,1)$-cycles to singular varieties.

Theorem 1.1 (Theorem 5.4 and Remark 6.2; cf. [Ara16, Theorem 7.8]). Suppose X 
is an arbitrary separated scheme $X$ of finite type over $\mathbb{C}$. Then, there are isomorphisms

$$
H_{c, c d h}^{2}\left(X, \mathbb{Z}(1)_{c d h}^{S F}\right)_{d i v} \underset{\text { canonical }}{\cong} H_{c, L, h o m}^{2}(X, \mathbb{Z}(1)) \underset{A J_{c, L}^{2,1}}{\stackrel{\cong}{\longrightarrow}} J_{c}^{2,1}(X)
$$

and a surjection

$$
H_{c, c d h}^{2}\left(X, \mathbb{Z}(1)_{c d h}^{S F}\right) \underset{\text { canonical }}{\cong} H_{c, L}^{2}(X, \mathbb{Z}(1)) \stackrel{c l_{c, L}^{B}}{\longrightarrow} H_{c, B}^{2}(X, \mathbb{Z}(1)) \cap F^{1} H_{c, B}^{2}(X, \mathbb{C}),
$$

where $H_{c, c d h}^{2}\left(X, \mathbb{Z}(1)_{c d h}^{S F}\right)_{d i v}$ is the maximal divisible subgroup of $H_{c, c d h}^{2}\left(X, \mathbb{Z}(1)_{c d h}^{S F}\right)$.

For other degrees $(m, n)$, we show that the cycle maps $c l_{c, L}^{\mathcal{D B}}$ and the version $c l_{L}^{\mathcal{D B}}$ without compact supports are always surjective on torsion and have torsion-free cokernels (Theorem 6.4 (i)). We shall also prove the following.

Theorem 1.2 (Theorem 6.4 and Corollary 6.5; cf. [RS16, Proposition 5.1] for the smooth projective case). For an arbitrary separated scheme of finite type over $\mathbb{C}$ and non-negative integers $m$ and $n$,

$i$. If $m \leqslant 2 n$, then the maps $c l_{c, L}^{\mathcal{D B}}$ and $A J_{c, L}^{m, n}$ are isomorphisms on torsion.

ii. If $\min \{2 m-1,2 \operatorname{dim} X+1\} \leqslant 2 n$, then the maps $c_{L}^{\mathcal{D B}}$ and $A J_{L}^{m, n}$ are isomorphisms on torsion.

iii. If $m<2 n$, then im $\left(c l_{c, L}^{B}\right)=H_{c, B}^{m}(X, \mathbb{Z}(n)) \cap F^{n} H_{c, B}^{m}(X, \mathbb{C})=H_{c, B}^{m}(X, \mathbb{Z}(n))_{\text {tor }}$.

iv. If $\min \{2 m, 2 \operatorname{dim} X\}<2 n$, then $i m\left(c l_{L}^{B}\right)=H_{B}^{m}(X, \mathbb{Z}(n)) \cap F^{n} H_{B}^{m}(X, \mathbb{C})=H_{B}^{m}$ $(X, \mathbb{Z}(n))_{\text {tor }}$.

Krishna and Pelaez [KP17, Theorem 1.5] proved a similar result for projective schemes in the zero cycle case (i.e. when $m=2 n=2 \operatorname{dim} X$ ). They used Voevodsky's motivic cohomology, not Lichtenbaum cohomology, and constructed the Abel-Jacobi maps via $K$-theory. See Remark 6.6 for more on the relation with their work.

With Theorem 1.2, or by the smooth projective case already known by the work of Rosenschon and Srinivas, we may characterize the "algebraic part" of Griffiths's intermediate Jacobians by a universal property. This characterization is algebraic, and as a variant of [Gei17], it makes sense over any algebraically closed field.

In Section 2, we give a detailed proof of the étale descent for Deligne-Beilinson cohomology and other cohomology theories of our interest. In Section 3, we prove the eh descent for these cohomologies. This enables us to define Deligne-Beilinson cohomology (with compact supports) for arbitrary complex algebraic varieties. After checking that our generalization behaves well with respect to mixed Hodge structures in a certain sense in Section 4, we proceed to define Deligne-Beilinson cycle maps for Lichtenbaum cohomology (with compact supports) of arbitrary complex varieties in Section 5. At this point, our versions of the Abel-Jacobi theorem and the Lefschetz theorem for singular varieties follow immediately. In Section 6, we study the torsion part of the Deligne-Beilinson cycle maps, and, finally, in Section 7, we discuss the universal property of Griffiths's intermediate Jacobians.

\section{Convention}

Unless otherwise noted, schemes are assumed to be separated and of finite type over the field $\mathbb{C}$ of complex numbers. The category of all schemes (resp., all smooth 
schemes) is denoted by $S c h / \mathbb{C}($ resp., $S m / \mathbb{C})$.

We simultaneously deal with cohomology with and without compact supports by using a subscript $(c)$; for example, see Corollary 3.4 and the diagram (17). This subscript always means that the statements hold respectively for compactly supported theory and non-compactly supported theory.

\section{Acknowledgments}

We would like to thank the referee and the editor for helpful suggestions. The author would also like to thank Thomas Geisser and Shane Kelly for helpful conversations.

\section{Preliminaries: Deligne-Beilinson cohomology}

We give a detailed proof of the étale descent property of Deligne-Beilinson cohomology while organizing relevant topics (contained in [Bei84, EV88]) for our purpose. We call a smooth compactification of a smooth scheme a good compactification if the boundary divisor is strict normal crossing.

Let $M f d / \mathbb{C}$ be the category of complex manifolds and let $h: S m / \mathbb{C} \rightarrow M f d / \mathbb{C}$ be the complex analytification functor. By a complex of analytic sheaves $\mathcal{F}^{\bullet}$ on a smooth scheme $X$ (over $\mathbb{C}$ ), we mean a complex of sheaves $\mathcal{F}$ on the underlying topological space of $h(X)$. We call the site consisting of open subsets of $h(X)$ with jointly surjective families of inclusions the analytic site of $X$ and write it as $X_{a n}$. The sheaf hypercohomology $H^{m}\left(h(X), \mathcal{F}^{\bullet}\right)$ is called analytic hypercohomology of $X$ and written as $H_{a n}^{m}\left(X, \mathcal{F}^{\bullet}\right)$.

Definition 2.1 ([Bei84, EV88]). Let $X$ be a smooth scheme, and let $X \stackrel{j}{\hookrightarrow} \bar{X}$ be a good compactification with the boundary divisor $Z$. The Deligne-Beilinson cohomology of $X$ is defined as analytic hypercohomology

$$
H_{\mathcal{D B}}^{m}(X, \mathbb{Z}(n)):=H_{a n}^{m}\left(\bar{X}, \mathbb{Z}(n)_{\bar{X}, Z}^{\mathcal{D} \mathcal{B}}\right),
$$

where

$$
\mathbb{Z}(n)_{\bar{X}, Z}^{\mathcal{D B}}:=\operatorname{cone}\left(R j_{*} \underline{\mathbb{Z}(n)} \oplus \Omega_{\bar{X}}^{\bullet \geqslant n}(\log Z) \stackrel{\epsilon-\iota}{\longrightarrow} R j_{*} \Omega_{X}^{\bullet}\right)[-1]
$$

is a complex of analytic sheaves on $\bar{X}$. Here, $\mathbb{Z}(n)$ is the constant sheaf of value $(2 \pi i)^{n} \mathbb{Z}$, the complex $\Omega_{X}^{\bullet}$ stands for the de Rham complex of holomorphic forms on $X$ and $\Omega_{\bar{X}}^{\bullet \geqslant n}(\log Z)$ is the brutal truncation of the complex of meromorphic differential forms on $\bar{X}$ with at most logarithmic poles along $Z$. The maps $\epsilon$ and $\iota$ are the canonical ones.

As the notation suggests, Definition 2.1 is independent of the choice of the good compactification $\bar{X}$ [EV88, Lemma 2.8]. We may actually express the Deligne-Beilinson cohomology of a smooth scheme $X$ as hypercohomology of $X$ itself with coefficients in a complex of sheaves on the big Zariski site $(S m / \mathbb{C})_{Z a r}$. Let us recall the construction due to Beilinson [Bei84, 1.6.5] (see also [EV88, Sections 4 and 5]).

Consider the category $\Pi$ whose objects are good compactifications $j: T \hookrightarrow \bar{T}$ ( $T$ is any smooth scheme) and morphisms are given by the usual commutative diagrams. 
We would like to define a suitable notion of analytic sheaves on $\Pi$. For motivating examples, see Definition 2.3.

We start by defining an analytic sheaf on a good compactification $j: T \hookrightarrow \bar{T}$ as a pair of analytic sheaves $\mathcal{F}$ on $T$ and $\overline{\mathcal{F}}$ on $\bar{T}$ (i.e. sheaves of abelian groups on the small analytic sites $T_{a n}$ and $\left.\bar{T}_{a n}\right)$ together with a sheaf morphism $\phi: \overline{\mathcal{F}}_{\rightarrow} j_{*} \mathcal{F}$. The analytic sheaves on good compactification $j: T \hookrightarrow \bar{T}$ form the category $\operatorname{Sh}_{a n}(\bar{T}, T)$ with morphisms pairs $\left(\bar{f}: \overline{\mathcal{F}} \rightarrow \overline{\mathcal{F}}^{\prime}, f: \mathcal{F} \rightarrow \mathcal{F}^{\prime}\right)$ of maps of analytic sheaves that make the diagram

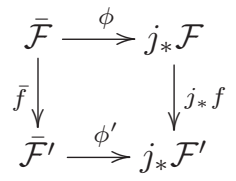

commute.

The category $S h_{a n}(\bar{T}, T)$ has enough injectives. Indeed, if $\bar{i}: \overline{\mathcal{F}} \hookrightarrow \overline{\mathcal{I}}$ and $i: \mathcal{F} \hookrightarrow \mathcal{I}$ are imbeddings into injective sheaves, the map

$$
\left(\bar{i} \oplus\left(j_{*}(i) \circ \phi\right), i\right):(\overline{\mathcal{F}}, \mathcal{F}, \phi) \longrightarrow\left(\overline{\mathcal{I}} \oplus j_{*} \mathcal{I}, \mathcal{I}, p r_{2}\right)
$$

is an imbedding into an injective object in $S h_{a n}(\bar{T}, T)[\mathbf{E V 8 8}, 4.2]$. In particular, if $\overline{\mathcal{F}} \rightarrow\left(\overline{\mathcal{I}}^{\bullet}, \bar{d}\right)$ and $\mathcal{F} \rightarrow\left(\mathcal{I}^{\bullet}, d\right)$ are injective resolutions, then

$$
(\overline{\mathcal{F}}, 0,0) \longrightarrow\left(\overline{\mathcal{I}}^{\bullet}, 0,0\right)
$$

and

$$
\begin{aligned}
&(0, \mathcal{F}, 0) \longrightarrow\left(j_{*} \mathcal{I}^{0}, \mathcal{I}^{0}, i d\right) \stackrel{\delta^{0}}{\longrightarrow}\left(j_{*} \mathcal{I}^{0} \oplus j_{*} \mathcal{I}^{1}, \mathcal{I}^{1}, p r_{2}\right) \stackrel{\delta^{1}}{\longrightarrow}\left(j_{*} \mathcal{I}^{1} \oplus j_{*} \mathcal{I}^{2}, \mathcal{I}^{2}, p r_{2}\right) \\
& \stackrel{\delta^{2}}{\longrightarrow}\left(j_{*} \mathcal{I}^{2} \oplus j_{*} \mathcal{I}^{3}, \mathcal{I}^{3}, p r_{2}\right) \longrightarrow \cdots
\end{aligned}
$$

are injective resolutions in $S h_{a n}(\bar{T}, T)$. Here, the differential $\delta^{n}$ sends

$$
(x \oplus y, z) \in\left(j_{*} \mathcal{I}^{n-1} \oplus j_{*} \mathcal{I}^{n}, \mathcal{I}^{n}, p r_{2}\right)
$$

to

$$
\left(-j_{*}(d)(x)+y \oplus j_{*}(d)(y), d(z)\right) \in\left(j_{*} \mathcal{I}^{n} \oplus j_{*} \mathcal{I}^{n+1}, \mathcal{I}^{n+1}, p r_{2}\right) .
$$

Note that these descriptions are still valid even when $\overline{\mathcal{F}}$ and $\mathcal{F}$ are replaced with complexes of sheaves $\overline{\mathcal{F}}^{\bullet}$ and $\mathcal{F}^{\bullet}$ and $\overline{\mathcal{I}}^{\bullet}$ and $\mathcal{I}^{\bullet}$ with their injective resolutions; i.e.

$$
\left(\overline{\mathcal{F}}^{\bullet}, 0,0\right) \longrightarrow\left(\overline{\mathcal{I}}^{\bullet}, 0,0\right)
$$

and

$$
\left(0, \mathcal{F}^{\bullet}, 0\right) \longrightarrow\left(j_{*} \mathcal{I}^{\bullet}[-1] \oplus j_{*} \mathcal{I}^{\bullet}, \mathcal{I}^{\bullet}, p r_{2}\right)
$$

are quasi-isomorphisms.

A collection of analytic sheaves $\left\{\left(\overline{\mathcal{F}}_{\bar{T}}, \mathcal{F}_{T}, \phi_{j}\right)\right\}_{j: T \hookrightarrow \bar{T}}$ on good compactifications $j: T \hookrightarrow \bar{T}$ together with a morphism $f^{\sharp}:\left(\overline{\mathcal{F}}_{\bar{T}^{\prime}}, \mathcal{F}_{T^{\prime}}, \phi_{j^{\prime}}\right) \rightarrow\left(\bar{f}_{*} \overline{\mathcal{F}}_{\bar{T}}, f_{*} \mathcal{F}_{T}, f_{*} \phi_{j}\right)$ for each morphism $(f, \bar{f}):(T \stackrel{j}{\hookrightarrow} \bar{T}) \rightarrow\left(T^{\prime} \stackrel{j^{\prime}}{\hookrightarrow} \bar{T}^{\prime}\right)$ in $\Pi$ such that $(f \circ g)^{\sharp}=g^{\sharp} \circ f^{\sharp}$ and $i d^{\sharp}=i d$ is called an analytic sheaf on $\Pi$. The category of analytic sheaves on $\Pi$ is written as $S h_{a n}(\Pi)$. Since analytic sheaves have functorial Godement injective imbeddings, the imbedding (2) can be chosen functorially with respect to the morphisms of $\Pi$. Thus, $S h_{a n}(\Pi)$ has enough injectives. 
Now, let $\tau \in\{$ triv, Zar, Nis, ét $\}$ (triv stands for the trivial topology) and consider the functor $\sigma_{\tau}: S h_{a n}(\Pi) \rightarrow S h_{\tau}(S m / \mathbb{C})$ that sends $\left\{\left(\overline{\mathcal{F}}_{\bar{T}}, \mathcal{F}_{T}, \phi_{j}\right)\right\}_{j: T \hookrightarrow \bar{T}}$ to the $\tau$ sheafification of the presheaf

$$
S m / \mathbb{C} \ni T \mapsto \operatorname{colim}_{j: T \hookrightarrow \bar{T}} \Gamma_{\bar{T}, T}\left(\overline{\mathcal{F}}_{\bar{T}}, \mathcal{F}_{T}, \phi_{j}\right),
$$

where the colimit is taken over all good compactifications of $T$ and $\Gamma_{\bar{T}, T}$ is the left exact functor $\Gamma_{\bar{T}, T}\left(\overline{\mathcal{F}}_{\bar{T}}, \mathcal{F}_{T}, \phi_{j}\right)=\operatorname{ker}\left\{\overline{\mathcal{F}}_{\bar{T}}(\bar{T}) \stackrel{\phi_{j}}{\rightarrow} \mathcal{F}_{T}(T)\right\}$. Since the colimit is taken over a directed system and sheafification is an exact functor, $\sigma_{\tau}$ is left exact. Deriving it, we obtain

$$
R \sigma_{\tau}: D^{+}\left(S h_{a n}(\Pi)\right) \rightarrow D^{+}\left(S h_{\tau}(S m / \mathbb{C})\right) .
$$

It is important for us that, given a complex of sheaves $\left\{\left(\overline{\mathcal{F}}_{\bar{T}}^{\bullet}, \mathcal{F}_{T}^{\bullet}, \phi_{j}^{\bullet}\right)\right\}_{j: T \hookrightarrow \bar{T}}$ in $S h_{a n}(\Pi)$, there is always a canonical map of presheaves

$$
R \sigma_{\text {triv }}\left(\left\{\left(\overline{\mathcal{F}}_{\bar{T}}^{\bullet}, \mathcal{F}_{T}^{\bullet}, \phi_{j}^{\bullet}\right)\right\}_{j: T \hookrightarrow \bar{T}}\right) \stackrel{\text { sheafification }}{\longrightarrow} R \sigma_{\tau}\left(\left\{\left(\overline{\mathcal{F}}_{\bar{T}}^{\bullet}, \mathcal{F}_{T}^{\bullet}, \phi_{j}^{\bullet}\right)\right\}_{j: T \hookrightarrow \bar{T}}\right) .
$$

For each $X \in S m / \mathbb{C}$, it induces a homomorphism

$$
H^{m}\left(R \sigma_{\text {triv }}\left(\left\{\left(\overline{\mathcal{F}}_{\bar{T}}^{\bullet}, \mathcal{F}_{T}^{\bullet}, \phi_{j}^{\bullet}\right)\right\}_{j: T \hookrightarrow \bar{T}}\right)(X)\right) \longrightarrow H_{\tau}^{m}\left(X, R \sigma_{\tau}\left(\left\{\left(\overline{\mathcal{F}}_{\bar{T}}^{\bullet}, \mathcal{F}_{T}^{\bullet}, \phi_{j}^{\bullet}\right)\right\}_{j: T \hookrightarrow \bar{T}}\right)\right) .
$$

By definition, the question whether the map (5) is an isomorphism for all $m$ is a question if the restriction of the complex of presheaves $R \sigma_{\operatorname{triv}}\left(\left\{\left(\overline{\mathcal{F}}_{\bar{T}}^{\bullet}, \mathcal{F}_{T}^{\bullet}, \phi_{j}^{\bullet}\right)\right\}_{j: T \hookrightarrow \bar{T}}\right)$ to the small site $X_{\tau}$ satisfies $\tau$-descent.

It is convenient to have the following lemma.

Lemma 2.2 ([EV88, Proposition $4.4($ a) $])$. For any complex $\left\{\left(\overline{\mathcal{F}}_{\bar{T}}^{\bullet}, \mathcal{F}_{T}^{\bullet}, \phi_{j}^{\bullet}\right)\right\}_{j: T \hookrightarrow \bar{T}}$ of analytic sheaves on $\Pi$, we have

$$
\begin{aligned}
& R \sigma_{\tau}\left(\left\{\left(\overline{\mathcal{F}}_{\bar{T}}^{\bullet}, \mathcal{F}_{T}^{\bullet}, \phi_{j}^{\bullet}\right)\right\}_{j: T \hookrightarrow \bar{T}}\right) \\
& \quad=\operatorname{cone}\left(R \sigma_{\tau}\left(\left\{\left(\overline{\mathcal{F}}_{\bar{T}}^{\bullet}, 0,0\right)\right\}_{j: T \hookrightarrow \bar{T}} \stackrel{R \sigma_{\tau}\left(\phi_{j}^{\bullet}\right)}{\longrightarrow} R \sigma_{\tau}\left(\left\{\left(0, \mathcal{F}_{T}^{\bullet}[1], 0\right)\right\}_{j: T \hookrightarrow \bar{T}}\right)\right)[-1]\right.
\end{aligned}
$$

Proof. It is enough to observe the identity for injective sheaves of the form $\left\{\left(\overline{\mathcal{I}}_{\bar{T}} \oplus\right.\right.$ $\left.\left.j_{*} \mathcal{I}_{T}, \mathcal{I}_{T}, p r_{2}\right)\right\}_{j: T \hookrightarrow \bar{T}}$, where $\overline{\mathcal{I}}_{\bar{T}}$ (resp., $\mathcal{I}_{T}$ ) is an injective sheaf on $\bar{T}$ (resp., $T$ ) because every sheaf in $S h_{a n}(\Pi)$ can be imbedded into an injective sheaf of this form.

Definition 2.3. Consider the following complexes of sheaves:

i. $\left\{D B(n)_{\bar{T}, T}\right\}_{T \hookrightarrow \bar{T}}:=\left\{\left(\Omega_{\bar{T}}^{\bullet \geqslant n}(\log (\bar{T} \backslash T)), \operatorname{cone}\left(\underline{\mathbb{Z}(n)} \stackrel{\epsilon}{\rightarrow} \Omega_{T}^{\bullet}\right),-\iota\right)\right\}_{T \hookrightarrow \bar{T}}$.

ii. $\left\{D R_{\bar{T}, T}\right\}_{T \hookrightarrow \bar{T}}:=\left\{\left(0, \Omega_{T}^{\bullet}[1], 0\right)\right\}_{T \hookrightarrow \bar{T}}$.

iii. $\left\{D R_{\bar{T}, T}^{\geqslant n}\right\}_{T \hookrightarrow \bar{T}}:=\left\{\left(\Omega_{\bar{T}}^{\bullet \geqslant n}(\log (\bar{T} \backslash T)), 0,0\right)\right\}_{T \hookrightarrow \bar{T}}$.

iv. $\left\{D R_{\bar{T}, T}^{<n}\right\}_{T \hookrightarrow \bar{T}}:=\left\{\left(\Omega_{\bar{T}}^{\bullet \geqslant n}(\log (\bar{T} \backslash T))[1], \Omega_{T}^{\bullet}[1],-\iota\right)\right\}_{T \hookrightarrow \bar{T}}$.

v. $\left\{B(n)_{\bar{T}, T}\right\}_{T \hookrightarrow \bar{T}}:=\{(0, \mathbb{Z}(n)[1], 0)\}_{T \hookrightarrow \bar{T}}$.

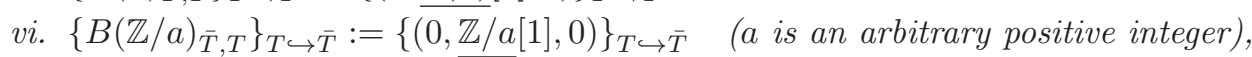
where, $\mathbb{Z}(n):=\underline{2 n \pi \mathbb{Z}}$ and $\mathbb{Z} / a$ denote the constant analytic sheaves. The respective

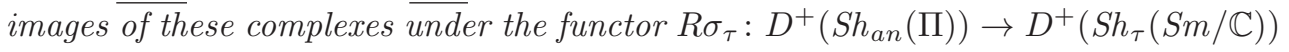
as: 

i. $D B(n)_{\tau}$,
ii. $D R_{\tau}$,
iii. $D R_{\tau}^{\geqslant n}$,
iv. $D R_{\tau}^{<n}$,
v. $B(n)_{\tau}$,
vi. $B(\mathbb{Z} / a)_{\tau}$.

Proposition 2.4. In $D^{+}\left(S h_{\tau}(S m / \mathbb{C})\right)$, there are distinguished triangles:

$$
\begin{gathered}
D R_{\tau}^{\geqslant n} \longrightarrow D R_{\tau} \longrightarrow D R_{\tau}^{<n} \stackrel{[+1]}{\longrightarrow}, \\
D R_{\tau}^{<n}[-1] \longrightarrow D B(n)_{\tau} \longrightarrow B(n)_{\tau} \stackrel{[+1]}{\longrightarrow}, \\
D B(n)_{\tau} \stackrel{\times a}{\longrightarrow} D B(n)_{\tau} \longrightarrow B(\mathbb{Z} / a)_{\tau} \stackrel{[+1]}{\longrightarrow},
\end{gathered}
$$

where $a$ is any positive integer.

Proof. This follows from Lemma 2.2.

Theorem 2.5 (Etale descent; cf. [Bei84, 1.6.5], [EV88, 5.5], [HS15, Theorem 2.8(vi)]). The complexes of presheaves $D B(n)_{\text {triv }}, D R_{\text {triv }}, D R_{\text {triv }}^{\geqslant n}, D R_{\text {triv }}^{<n}, B(n)_{\text {triv }}$, and $B(\mathbb{Z} / a)_{\text {triv }}$ satisfy étale descent on any $X \in S m / \mathbb{C}$; and for $\tau \in\{$ Zar, Nis, ét $\}$, the map (5) is identified, respectively, with

$$
\begin{aligned}
\text { i. } H_{\tau}^{m}\left(X, D B(n)_{\tau}\right) \cong H_{\mathcal{D B}}^{m}(X, \mathbb{Z}(n)), \\
\text { ii. } H_{\tau}^{m}\left(X, D R_{\tau}\right) \cong H_{d R}^{m}(X, \mathbb{C}), \\
\text { iii. } H_{\tau}^{m}\left(X, D R_{\tau}^{\geqslant n}\right) \cong H_{a n}^{m}\left(\bar{X}, \Omega_{\bar{X}}^{\bullet \geqslant n}(\log \bar{X} \backslash X)\right), \\
\text { iv. } H_{\tau}^{m}\left(X, D R_{\tau}^{<n}\right) \cong H_{d R}^{m}(X, \mathbb{C}) / F^{n} H_{d R}^{m}(X, \mathbb{C}), \\
\text { v. } H_{\tau}^{m}\left(X, B(n)_{\tau}\right) \cong H_{B}^{m}(X, \mathbb{Z}(n)), \\
\text { vi. } H_{\tau}^{m}\left(X, B(\mathbb{Z} / a)_{\tau}\right) \cong H_{B}^{m}(X, \mathbb{Z} / a) .
\end{aligned}
$$

In $i v, F^{n}$ signifies the $n$-th Hodge filtration.

Proof. Let us start with $i i, v$ and $v i$. Let $c l$ denote the topology on the category $M f d / \mathbb{C}$ of complex manifolds generated by jointly surjective families of local isomorphisms. Under complex analytification $h: S m / \mathbb{C} \rightarrow M f d / \mathbb{C}$, any $\tau$-cover induces a $c l$-cover $\left[\right.$ Art73, (4.0)]. Suppose $\mathcal{F}^{\bullet}$ is a complex of sheaves on $(M f d / \mathbb{C})_{a n}$, where $(M f d / \mathbb{C})_{a n}$ is the site whose covers are by definition jointly surjective families of inclusions of open submanifolds. (For $i i, \mathcal{F}^{\bullet}$ is $\Omega^{\bullet}[1]$, and for $v$ and $i v$, it is $\mathbb{Z}(n)[1]$ and $\mathbb{Z} / a[1]$, respectively; see Definition 2.3.) Since any $c l$-cover has a refinement by an an-cover, we have an equality of topoi $S h_{a n}(M f d / \mathbb{C})=S h_{c l}(M f d / \mathbb{C})$. Let $\mathcal{I}^{\bullet}$ be an injective resolution of $\mathcal{F}^{\bullet}$ in this topos.

Now, let $\mathcal{F}_{T}^{\bullet}$ be the restriction of $\mathcal{F}^{\bullet}$ to $T_{a n}(T \in S m / \mathbb{C})$ (i.e. the restriction to the underlying topological space of $h(T) \in M f d / \mathbb{C}$ ) and consider the complex 
$\left\{\left(0, \mathcal{F}_{T}^{\bullet}, 0\right)\right\}_{T \hookrightarrow \bar{T}}$ of analytic sheaves on $\Pi$. By the explicit description (3), we have

$$
R \sigma_{\tau}\left(\left\{\left(0, \mathcal{F}_{T}^{\bullet}, 0\right)\right\}_{T \hookrightarrow \bar{T}}\right)=\left(\mathcal{I}^{\bullet} \circ h\right)[-1],
$$

where, on the right hand side, the complex of sheaves $\mathcal{I}^{\bullet}$ on $(M f d / \mathbb{C})_{c l}$ is regarded as a complex of sheaves on $(S m / \mathbb{C})_{\tau}$ by pulling it back along the complex analytification functor $h$. For any $X \in S m / \mathbb{C}$, we have

$$
\begin{aligned}
H_{\tau}^{m}\left(X, R \sigma_{\tau}\left(\left\{\left(0, \mathcal{F}_{T}^{\bullet}, 0\right)\right\}_{T \hookrightarrow \bar{T}}\right)\right) & =H_{\tau}^{m}\left(X,\left(\mathcal{I}^{\bullet} \circ h\right)[-1]\right) \\
& =H_{\tau}^{m}\left(X,\left(\mathcal{I}^{\bullet} \circ h\right)_{\left.\right|_{X_{\tau}}}[-1]\right) \\
& =H^{m}\left(\left(\mathcal{I}^{\bullet} \circ h(X)\right)[-1]\right) .
\end{aligned}
$$

Thus, the isomorphisms $i i, v$ and $v i$ follow. Here, the last equality requires an explanation. It holds if $\left(\mathcal{I}^{\bullet} \circ h\right)_{X_{\tau}}$, the restriction of $\mathcal{I}^{\bullet} \circ h$ to the small $\tau$-site $X_{\tau}$, is a complex of injective sheaves. Let us prove this. Instead of pulling back $\mathcal{I}^{\bullet}$ along $h$ and restricting to $X_{\tau}$, the complex $\left(\mathcal{I}^{\bullet} \circ h\right)_{\left.\right|_{\tau}}$ can also be obtained by first pulling back $\mathcal{I}^{\bullet}$ from $(M f d / \mathbb{C})_{c l}$ to the small site $h(X)_{c l}$, and then to $X_{\tau}$ along the morphism $X_{\tau} \rightarrow h(X)_{c l}$ between the underlying categories induced by $h$. Both of these restriction functors have left adjoint functors (obtained by applying sheafification to left Kan extensions; see [Mac98, Chapter X, Section 3; esp. (10)]) and they are exact because the underlying categories of $h(X)_{c l}$ and $X_{\tau}$ have finite limits and hence the colimit in the explicit description of Kan extensions in loc. cit. is directed; cf. the proof of [Mil80, Chapter 2, Proposition 2.6]. Hence, the two restriction functors in question preserve injective objects.

For the remaining $i, i i i$ and $i v, i$ and $i v$ follow from $i i i$ and the already proven $i i$, $v$ and $v i$. Let us explain how $i v$ follows. For any smooth scheme $X$, the distinguished triangle $(6)$ in $D^{+}\left(S h_{\tau}(S m / \mathbb{C})\right)$ and the map (5) give rise to the commutative diagram with exact rows

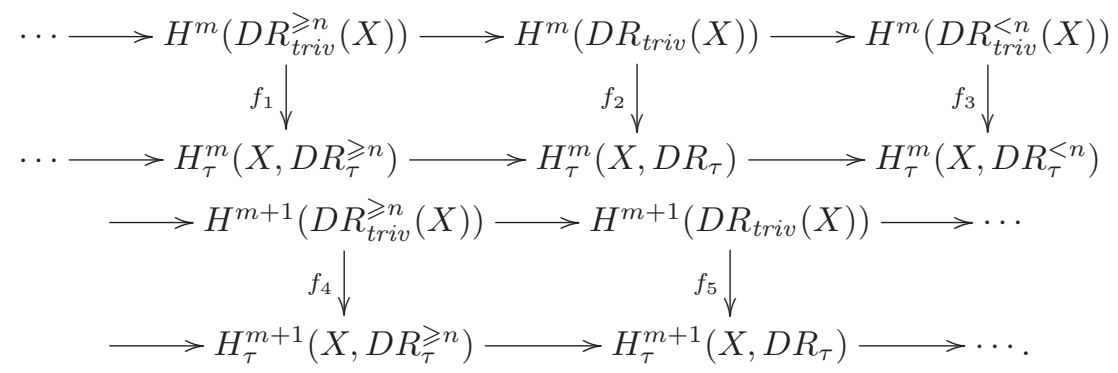

Note that

$$
\begin{aligned}
H^{m}\left(D R_{\text {triv }}^{\geqslant n}(X)\right) & =R^{m}\left(\operatorname{colim} \Gamma_{\bar{X}, X}\right)\left(\left\{D R_{\bar{T}, T}^{\geqslant n}\right\}_{T \hookrightarrow \bar{T}}\right) \\
& \cong \operatorname{colim} H_{a n}^{m}\left(\bar{X}, \Omega_{\bar{X}}^{\bullet \geqslant n}(\log \bar{X} \backslash X)\right) \cong H_{a n}^{m}\left(\bar{X}, \Omega_{\bar{X}}^{\bullet \geqslant n}(\log \bar{X} \backslash X)\right)
\end{aligned}
$$

by the independence of the Hodge filtration from the choice of a good compactification [Del71, Théorème 3.2.5]. Also, since the canonical map $H_{a n}^{i}\left(\bar{X}, \Omega_{\bar{X}}^{\bullet \geqslant n}(\log \bar{X} \backslash X)\right) \rightarrow$ $H_{a n}^{i}\left(\bar{X}, \Omega_{\bar{X}}^{\bullet}(\log \bar{X} \backslash X)\right)$ is injective for all $i[\operatorname{Del74}$, Scholie 8.1.9 (v)], we have isomorphisms

$$
H^{m}\left(D R_{\text {triv }}^{<n}(X)\right) \cong H^{m}\left(D R_{\text {triv }}(X)\right) / H^{m}\left(D R_{\text {triv }}^{\geqslant n}(X)\right) \cong H_{d R}^{m}(X, \mathbb{C}) / F^{n} H_{d R}^{m}(X, \mathbb{C}) .
$$

Now, $i v$ follows from $i$ and $i i i$ by the 5-lemma. The isomorphism $i$ can also be 
obtained from $i v$ and $v$ by a similar argument with the triangle (7).

Thus, it remains to prove $i i i$, or that the complex $D R_{\text {triv }}^{\geqslant n}$ of presheaves, when restricted to the small $\tau$-site on each $X \in S m / \mathbb{C}$, satisfies $\tau$-descent. But, this follows from [CD12, Theorem 3.3.2 and 3.3.23], [Del74, Scholie 8.1.9 (v)] and the strict compatibility of morphisms of mixed Hodge structures [Del71, Théorème 2.3.5 (iii)] as explained in the proof of $[\mathbf{H S 1 5}$, Theorem 2.8(vi)].

\section{3. eh descent for Deligne-Beilinson cohomology}

We extend the definition of Deligne-Beilinson cohomology $H_{\mathcal{D} \mathcal{B}}^{m}(X, \mathbb{Z}(n))$ (Definition 2.1) to arbitrary (complex) schemes and also define its compactly supported version. This is done by interpreting Deligne-Beilinson cohomology as eh hypercohomology. (For eh cohomology, we refer the reader to [Gei06, Sections 2 and 3].) Our definition behaves reasonably with respect to the Hodge structure of Betti cohomology (see Proposition 3.7 and Section 4).

Let $\tau \in\{c d h, e h\}$. By cdh or eh sheafification, we always mean the left adjoint functor of the forgetful functor [FV00, Section 3]

$$
S h_{\tau}(S c h / k) \longrightarrow S h_{Z a r}(S m / k) .
$$

The argument of [Gei06, Theorem 3.6] proves the following theorem. See also [CD12, Theorem 3.3.8].

Theorem 3.1. Let $\mathcal{F}$ be a bounded below complex of étale sheaves on $S m / \mathbb{C}$. Suppose that any abstract blow-up square

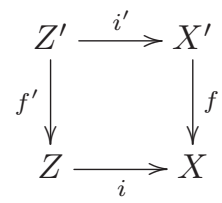

in $S m / \mathbb{C}$ gives rise to the distinguished triangle in the derived category of abelian groups

$$
R \Gamma_{X}(\mathcal{F}) \stackrel{\left(f^{*}, i^{*}\right)}{\longrightarrow} R \Gamma_{X^{\prime}}(\mathcal{F}) \oplus R \Gamma_{Z}(\mathcal{F}) \stackrel{i^{\prime *}-f^{\prime *}}{\longrightarrow} R \Gamma_{Z^{\prime}}(\mathcal{F}) \stackrel{[+1]}{\longrightarrow},
$$

where $R \Gamma_{S}$ is the right derived functor of the section functor over $S$.

Then, the canonical map

$$
H_{e ́ t}^{m}(X, \mathcal{F}) \longrightarrow H_{e h}^{m}\left(X, \mathcal{F}_{e h}\right)
$$

is an isomorphism for all $m$. A similar statement holds for the Nisnevich and cdh topologies as well.

Proof. We prove the theorem for the eh topology. The proof for the cdh topology is verbatim.

Let $C^{\bullet}:=\operatorname{cone}\left(\mathcal{F} \rightarrow R o_{*} \mathcal{F}_{e h}\right)$, where $o$ is the forgetful functor. We need to show that $H_{e ́ t}^{i}\left(X, C^{\bullet}\right)$ vanishes for all smooth schemes $X$ and $i \in \mathbb{Z}$. Suppose it is not true and there is some $X$ and $i$ for which the cohomology group $H_{e t}^{i}\left(X, C^{\bullet}\right)$ is non-trivial. First choose the smallest such integer $i$ when $X$ varies over all smooth schemes, and 
then choose $X$ of the smallest dimension for which $H_{e t}^{i}\left(X, C^{\bullet}\right)$ is non-trivial. Let $u$ be a non-zero element of this group.

Since the eh sheafification of $C^{\bullet}$ is acyclic, there is an eh cover $T \rightarrow X$ such that the image of $u$ under the homomorphism $H_{e ́ t}^{i}\left(X, C^{\bullet}\right) \rightarrow H_{e t t}^{i}\left(T, C^{\bullet}\right)$ vanishes. Now, the eh cover $T \rightarrow X$ is refined by a composition $U \stackrel{f}{\rightarrow} X^{\prime} \stackrel{b}{\rightarrow} X$, where $b$ is a series of blow-ups along smooth centers and $f$ is an étale cover [Gei06, Proposition 2.3]. We claim that the image of $u$ in $H_{e ́ t}^{i}\left(X^{\prime}, C^{\bullet}\right)$ is non-zero. We may obviously assume that the map $b$ consists of a single blow-up, say

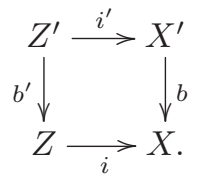

Let $\mathcal{F} \rightarrow \mathcal{I}^{\bullet}$ and $\mathcal{F}_{e h} \rightarrow \mathcal{J}^{\bullet}$ be injective resolutions in the respective topoi. Then, since $R o_{*} \mathcal{F}_{\text {eh }}=\mathcal{J}^{\bullet}$ is still a complex of injective sheaves in the étale topology, the canonical map $C^{\bullet}=\operatorname{cone}\left(\mathcal{F} \rightarrow \mathcal{J}^{\bullet}\right) \rightarrow \operatorname{cone}\left(\mathcal{I}^{\bullet} \rightarrow \mathcal{J}^{\bullet}\right)=: \mathcal{K}^{\bullet}$ is an injective resolution of $C^{\bullet}$.

Since the abstract blow-up square (9) gives rise to distinguished triangles for $\mathcal{I}^{\bullet}$ by the assumption of the theorem and for $\mathcal{J}^{\bullet}$ because an abstract blow-up square is an eh cover, we obtain the distinguished triangle

$$
\mathcal{K} \bullet(X) \stackrel{\left(b^{*}, i^{*}\right)}{\longrightarrow} \mathcal{K} \bullet\left(X^{\prime}\right) \oplus \mathcal{K} \bullet(Z) \stackrel{i^{\prime *}-b^{\prime *}}{\longrightarrow} \mathcal{K} \bullet\left(Z^{\prime}\right) \stackrel{[+1]}{\longrightarrow}
$$

by the octahedral axiom. Taking the cohomology groups, we get the long exact sequence

$$
\longrightarrow H_{e ́ t}^{i-1}\left(Z^{\prime}, C^{\bullet}\right) \longrightarrow H_{e ́ t}^{i}\left(X, C^{\bullet}\right) \stackrel{\left(b^{*}, i^{*}\right)}{\longrightarrow} H_{e ́ t}^{i}\left(X^{\prime}, C^{\bullet}\right) \oplus H_{e ́ t}^{i}\left(Z, C^{\bullet}\right) \longrightarrow .
$$

By our choice of $i$ and $X$, the cohomology groups of $Z$ and $Z^{\prime}$ of degree less than or equal to $i$ vanish. Therefore, the map

$$
b^{*}: H_{e ́ t}^{i}\left(X, C^{\bullet}\right) \longrightarrow H_{e ́ t}^{i}\left(X^{\prime}, C^{\bullet}\right)
$$

is injective. Hence, the image $u^{\prime}$ of $u$ in $H_{e ́ t}^{i}\left(X^{\prime}, C^{\bullet}\right)$ is non-zero.

Now, consider the spectral sequence

$$
E_{1}^{p, q}=H_{e ́ t}^{q}\left(U \times_{X^{\prime}} \cdots \times_{X^{\prime}} U, C^{\bullet}\right) \Longrightarrow H_{e ́ t}^{p+q}\left(X^{\prime}, C^{\bullet}\right) .
$$

By our choice of $i, E_{1}^{p, q} \cong H_{e t t}^{q}\left(U \times_{X^{\prime}} \cdots \times_{X^{\prime}} U, C^{\bullet}\right)=0$ if $q<i$. Thus, the canonical map $H_{e ́ t}^{i}\left(X^{\prime}, C^{\bullet}\right) \rightarrow H_{e ́ t}^{i}\left(U, C^{\bullet}\right)$ is injective, which means that the image of $u^{\prime}$ is nonzero. This is a contradiction because $b \circ f: U \rightarrow X$ factors through the eh cover $T \rightarrow$ $X$ on which $u$ vanishes.

Corollary 3.2. Let $\mathcal{F} \in\left\{B D(n)_{Z a r}, D R_{Z a r}, D R_{Z a r}^{\geqslant n}, D R_{Z a r}^{<n}, B(n)_{Z a r}, B(\mathbb{Z} / a)_{Z a r}\right\}$. Then, the canonical maps

$$
H_{Z a r}^{m}(X, \mathcal{F}) \longrightarrow H_{c d h}^{m}\left(X, \mathcal{F}_{c d h}\right) \longrightarrow H_{e h}^{m}\left(X, \mathcal{F}_{e h}\right)
$$

are isomorphisms for any $X \in S m / \mathbb{C}$.

Proof. By Theorem 2.5, it suffices to observe that the complexes $\mathcal{F}_{\text {Nis }}$ of Nisnevich sheaves have distinguished triangles associated with abstract blow-up squares of 
smooth schemes as in Theorem 3.1. By Proposition 2.4, it suffices to check this for $\mathcal{F}_{N i s} \in\left\{D R_{N i s}, B(n)_{N i s}, B(\mathbb{Z} / a)_{N i s}, D R_{N i s}^{\geqslant n}\right\}$. We need to show the excision property, i.e. the homomorphism $H_{N i s}^{m}\left(X, Z, \mathcal{F}_{N i s}\right) \stackrel{f^{*}}{\rightarrow} H_{N i s}^{m}\left(X^{\prime}, Z^{\prime}, \mathcal{F}_{N i s}\right)$ (notation as in Theorem 3.1) between relative Nisnevich cohomology groups is an isomorphism for all $m$. By relative Nisnevich cohomology $H_{N i s}^{m}\left(X, Z, \mathcal{F}_{N i s}\right)$, we simply mean the cohomology group $H_{N i s}^{m}\left(\operatorname{cone}\left(\mathcal{I}^{\bullet}(X) \rightarrow \mathcal{I}^{\bullet}(Z)[-1]\right)\right)$, where $\mathcal{I}^{\bullet}$ is an injective resolution of $\mathcal{F}$.

The excision property for the first three cases of Betti and de Rham cohomology follows from the proper base change theorem as explained in [HM-S17, Proposition 2.1.8]. The case $\mathcal{F}=D R_{N i s}^{\geqslant n}$ follows from the excision property for de Rham cohomology and the strict compatibility of morphisms of mixed Hodge structures [Del71, Théorème 2.3.5 (iii)].

Before stating the next corollary, let us agree on what we mean by eh (resp., cdh) cohomology with compact supports.

Definition 3.3 ([Gei06, Definition 3.3; see also Remark on p. 36]). Let $\mathcal{F}$ be a complex of eh (resp., cdh) sheaves on $S c h / k$. For any $X \in S c h / k$, the eh (resp., cdh) cohomology of $X$ with coefficients in $\mathcal{F}$ is defined as

$$
\begin{aligned}
H_{c, e h}^{m}(X, \mathcal{F}) & :=H^{m}(\operatorname{cone}(\mathcal{I}(\bar{X}) \longrightarrow \mathcal{I}(\bar{X} \backslash X))[-1]) \\
\left(\operatorname{resp} ., H_{c, c d h}^{m}(X, \mathcal{F})\right. & \left.:=H^{m}(\operatorname{cone}(\mathcal{J}(\bar{X}) \longrightarrow \mathcal{J}(\bar{X} \backslash X))[-1])\right),
\end{aligned}
$$

where $\bar{X}$ is a Nagata compactification of $X$ and $\mathcal{I}$ (resp., $\mathcal{J}$ ) is an injective resolution of $\mathcal{F}$ in the eh (resp., cdh) topology. of $\bar{X}$

It is proved in [Ibid., Lemma 3.4] that Definition 3.3 is independent of the choice

Corollary 3.4. For $\tau \in\{c d h, e h\}$ and any $X \in S c h / \mathbb{C}$, there are canonical isomorphisms of cohomology groups (resp., with compact supports):

$$
\begin{aligned}
\text { i. } & H_{(c), \tau}^{m}\left(X, D R_{\tau}\right) \cong H_{(c), B}^{m}(X, \mathbb{C}), \\
\text { ii. } & H_{(c), \tau}^{m}\left(X, B(n)_{\tau}\right) \cong H_{(c), B}^{m}(X, \mathbb{Z}(n)), \\
\text { iii. } & H_{(c), \tau}^{m}\left(X, B(\mathbb{Z} / a)_{\tau}\right) \cong H_{(c), B}^{m}(X, \mathbb{Z} / a) .
\end{aligned}
$$

Proof. Let us prove $(i)$ with compact supports for $\tau=c d h$. The other cases are similar. Let $X \hookrightarrow \bar{X}$ be a compactification with the boundary divisor $i: Z:=\bar{X} \backslash X \hookrightarrow \bar{X}$. Choose smooth proper cdh hypercovers $\epsilon: \bar{X}_{\bullet} \rightarrow \bar{X}$ and $\eta: Z \bullet \rightarrow Z$ with a commutative diagram (see $[$ Del74, (6.2.8)])

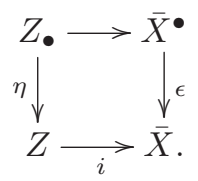

Let $\mathcal{I}^{\bullet}$ be an injective resolution of $\Omega^{\bullet}$ in $S h_{c l}(M f d / \mathbb{C})$. Then, as in the proof of Theorem 2.5, we have $D R_{c d h}=\mathcal{I}_{c d h}^{\bullet}$. (Here, the complex $\mathcal{I}_{c d h}^{\bullet}$ on the right hand side stands for the cdh sheafification of the complex of (pre)sheaves $\mathcal{I}^{\bullet} \circ h$ on $S m / \mathbb{C}$, where $h$ is the complex analytification functor $h: S m / \mathbb{C} \rightarrow M f d / \mathbb{C}$.) Let $D R_{c d h} \rightarrow \mathcal{J}^{\bullet}$ be an 
injective resolution in the cdh topology. Then, we have a sequence of isomorphisms

$$
\begin{aligned}
H_{c, c d h}^{m}\left(X, D R_{c d h}\right) & \stackrel{\text { def }}{=} H^{m}\left(\operatorname{cone}\left(\mathcal{J}^{\bullet}(\bar{X}) \longrightarrow \mathcal{J}^{\bullet}(Z)\right)[-1]\right) \\
\text { cone }\left(\epsilon^{*}, \eta^{*}\right) & H^{m}\left(\operatorname{cone}\left(\operatorname{Tot} \mathcal{J}^{\bullet}\left(\bar{X}_{\bullet}\right) \longrightarrow \operatorname{Tot} \mathcal{J}^{\bullet}\left(Z_{\bullet}\right)\right)[-1]\right) \\
\stackrel{\text { Cor. } 3.2}{\cong} & H^{m}\left(\operatorname{cone}\left(\operatorname{Tot} \mathcal{I}^{\bullet}\left(\bar{X}_{\bullet}\right) \longrightarrow \operatorname{Tot} \mathcal{I}^{\bullet}\left(Z_{\bullet}\right)\right)[-1]\right) \\
& \cong H_{\text {an }}^{m}\left(\bar{X}, \operatorname{cone}\left(\operatorname{R} \epsilon_{*} \Omega_{\bar{X}}^{\bullet} \longrightarrow i_{*} \Omega_{Z_{\bullet}}^{\bullet}[-1]\right)\right. \\
& \stackrel{(\text { a) }}{\cong} \\
& H_{c, B}^{m}(X, \mathbb{C}) .
\end{aligned}
$$

The isomorphism (a) is due to Deligne's cohomological descent [Del74, 5.3.5 (II), 5.3.6].

With Corollary 3.2, we can extend Definition 2.1.

Definition 3.5. The Deligne-Beilinson cohomology (resp., without compact supports) of an arbitrary scheme $X \in S c h / \mathbb{C}$ is defined as

$$
\begin{aligned}
H_{\mathcal{D B}}^{m}(X, \mathbb{Z}(n)) & :=H_{e h}^{m}\left(X, D B(n)_{e h}\right) \\
\left(\text { resp. }, H_{c, \mathcal{D B}}^{m}(X, \mathbb{Z}(n))\right. & \left.:=H_{c, e h}^{m}\left(X, D B(n)_{e h}\right)\right) .
\end{aligned}
$$

Remark 3.6. The use of eh hypercohomology instead of cdh one in Definition 3.5 is simply a matter of choice. Indeed, both eh and cdh hypercohomology groups can be calculated on smooth cdh hypercovers (by spectral sequences). Therefore, either way, Corollary 3.2 gives canonically isomorphic groups.

Deligne-Beilinson cohomology is related to Hodge filtrations and integral Betti cohomology by long exact sequences.

Proposition 3.7. For an arbitrary scheme $X$ over $\mathbb{C}$, there are long exact sequences

$$
\begin{aligned}
& \cdots \rightarrow H_{B}^{m-1}(X, \mathbb{C}) / F^{n} \rightarrow H_{\mathcal{D B}}^{m}(X, \mathbb{Z}(n)) \rightarrow H_{B}^{m}(X, \mathbb{Z}(n)) \rightarrow H_{B}^{m}(X, \mathbb{C}) / F^{n} \rightarrow \cdots, \\
& \cdots \rightarrow H_{c, B}^{m-1}(X, \mathbb{C}) / F^{n} \rightarrow H_{c, \mathcal{D B}}^{m}(X, \mathbb{Z}(n)) \rightarrow H_{c, B}^{m}(X, \mathbb{Z}(n))>H_{c, B}^{m}(X, \mathbb{C}) / F^{n} \rightarrow \cdots
\end{aligned}
$$

Proof. For any $X$, taking the eh hypercohomology with or without compact supports of the eh sheafification of the distinguished triangle (7) in Proposition 2.4, we obtain the long exact sequence of cohomology groups (resp., with compact supports)

$$
\begin{aligned}
\cdots \longrightarrow H_{(c), e h}^{m-1}\left(X, D R_{e h}^{<n}\right) & \longrightarrow H_{(c), \mathcal{D B}}^{m}(X, \mathbb{Z}(n)) \\
& \longrightarrow H_{(c), e h}^{m}\left(X, B(n)_{e h}\right) \longrightarrow H_{(c), e h}^{m}\left(X, D R_{e h}^{<n}\right) \longrightarrow \cdots
\end{aligned}
$$

Since $H_{(c), e h}^{m}\left(X, B(n)_{e h}\right)$ is isomorphic to $H_{(c), B}^{m}(X, \mathbb{Z}(n))$ by Corollary $3.4(i i)$, it suffices to show the isomorphisms $H_{(c), e h}^{m}\left(X, D R_{e h}^{<n}\right) \cong H_{(c), B}^{m}(X, \mathbb{C}) / F^{n}$ for all $m$.

With Corollary $3.4(i)$ and the long exact sequence for eh cohomology (with compact supports) associated with the eh sheafification of the distinguished triangle (6), it is enough to show that the canonical maps

$$
H_{c, e h}^{m}\left(X, D R_{e h}^{\geqslant n}\right) \stackrel{f}{\longrightarrow} H_{c, e h}^{m}\left(X, D R_{e h}\right) \cong H_{c, B}^{m}(X, \mathbb{C})
$$


and

$$
H_{e h}^{m}\left(X, D R_{e h}^{\geqslant n}\right) \stackrel{h}{\longrightarrow} H_{e h}^{m}\left(X, D R_{e h}\right) \cong H_{B}^{m}(X, \mathbb{C})
$$

are injective and their images are respectively nothing but the $n$-th Hodge filtration $F^{n}$ of $H_{c, s i n g}^{m}(X, \mathbb{C})$ and $H_{B}^{m}(X, \mathbb{C})$.

Let us first deal with the case with compact supports. Choose a diagram of the form (10) and injective resolutions $D R_{e h}^{\geqslant n} \rightarrow \mathcal{J}^{\bullet}$ and $D R_{e h} \rightarrow \mathcal{K}^{\bullet}$ in $S h_{e h}(S c h / \mathbb{C})$. Let us write the Godement (injective) resolution of an analytic sheaf $\mathcal{F}$ as $G d^{\bullet} \mathcal{F}$. Consider the diagram

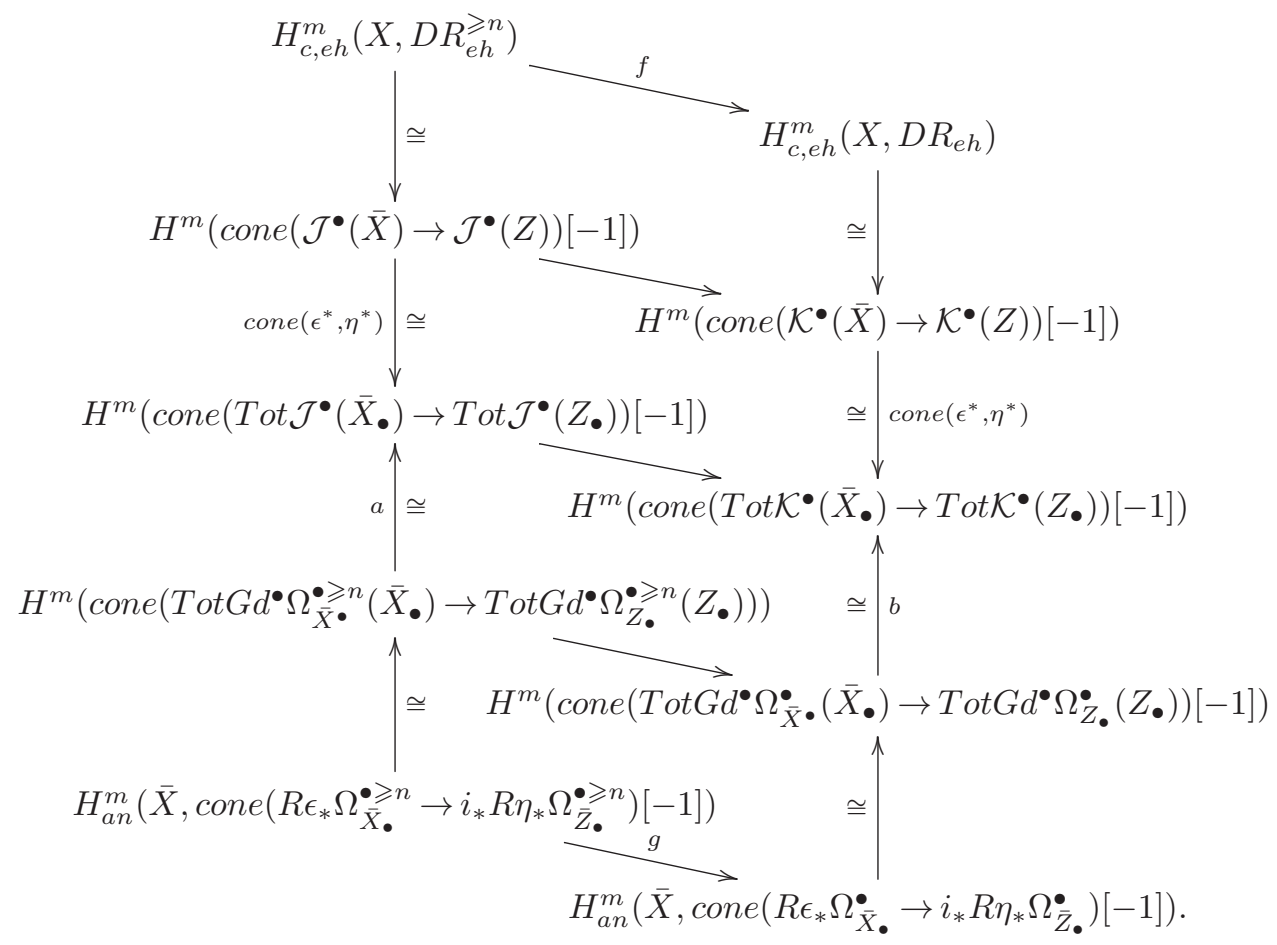

The maps $a$ and $b$ are the canonical ones induced by the map (4). (Recall that $D R_{e h}^{\geqslant n}$ (resp., $D R_{e h}$ ) is the eh sheafification of the complex of presheaves $S m / \mathbb{C} \ni U \mapsto$ $\operatorname{colim}_{U^{\text {good }} \breve{U}} T o t G d^{\bullet} \Omega_{\bar{U}}^{\bullet \geqslant n}(\log \bar{U} \backslash U)(\bar{U})$ (resp., $\left.U \mapsto G d^{\bullet} \Omega^{\bullet}(U)\right)$.) By the eh descent proved in Corollary 3.2, the maps $a$ and $b$ are isomorphisms. Therefore, the injectivity of $f$ and the desired property of its image follow because the map $g$ is injective by [Del74, Scholie $8.1 .9(\mathrm{v})]$ and its image in $H_{c, c d h}^{m}\left(X, D R_{c d h}\right) \cong H_{c, B}^{m}(X, \mathbb{C})$ is by definition $F^{n} H_{c, B}^{m}(X, \mathbb{C})$. This finishes the proof of (11).

Next, for the case without compact supports, choose a commutative diagram

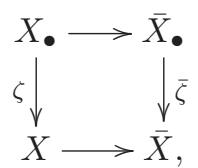

with smooth proper cdh hypercovers $\zeta$ and $\bar{\zeta}$ such that $X_{i} \rightarrow \bar{X}_{i}$ is a good compactifi- 
cation for each $i$. (For the existence of such hypercovers, see [Del74, (6.2.8)] and also the proof of [Con, Theorem 4.7] for details.) Then, we have a commutative diagram

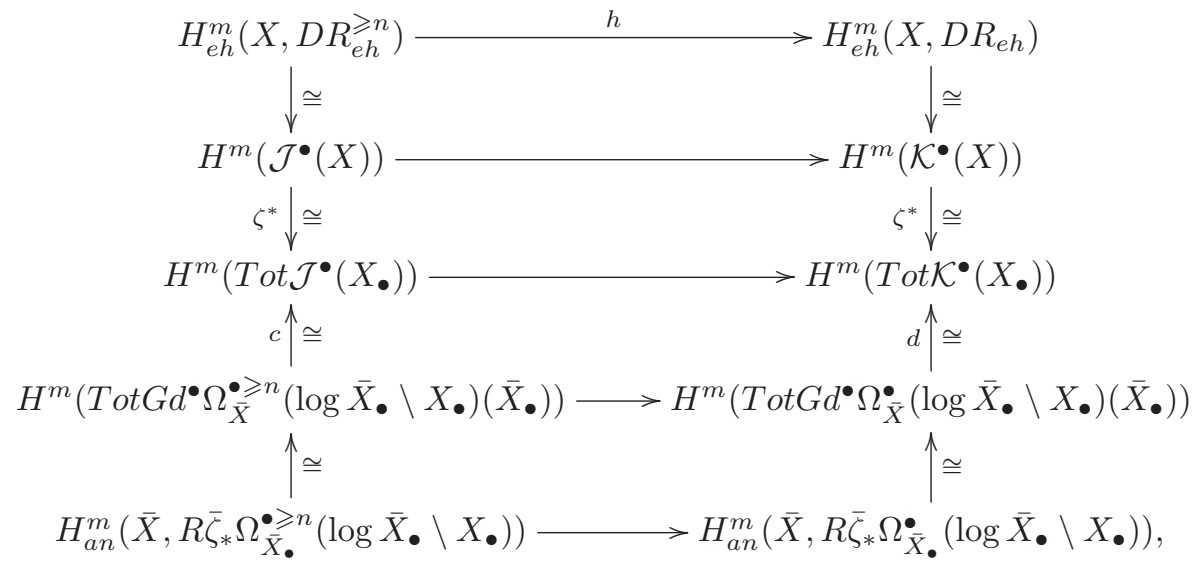

where $c$ and $d$ are the canonical maps as the maps $a$ and $b$, and they are isomorphisms by the proof of Corollary 3.2. The bottom map is injective by [Del74, Scholie 8.1.9 (v)] and its image is, by definition, the $n$-th Hodge filtration of $H_{B}^{m}(X, \mathbb{C})$.

Proposition 3.8. For any scheme $X \in S c h / \mathbb{C}$ and positive integer a, there is a long exact sequence of cohomology groups (resp., with compact supports)

$\rightarrow H_{(c), \mathcal{D} \mathcal{B}}^{m}(X, \mathbb{Z}(n)) \stackrel{\times a}{\longrightarrow} H_{(c), \mathcal{D} \mathcal{B}}^{m}(X, \mathbb{Z}(n)) \rightarrow H_{(c), B}^{m}(X, \mathbb{Z} / a) \rightarrow H_{(c), \mathcal{D} \mathcal{B}}^{m+1}(X, \mathbb{Z}(n)) \rightarrow$

Proof. Take the eh hypercohomology (resp., with compact supports) of the eh sheafification of the distinguished triangle (8) and apply Corollary 3.4 (iii).

\section{Intermediate Jacobians}

The short exact sequence for smooth proper $X[\mathbf{E V 8 8},(7.9)]$

$$
0 \longrightarrow J_{G}^{r}(X) \longrightarrow H_{\mathcal{D B}}^{2 r}(X, \mathbb{Z}(r)) \longrightarrow H d g^{r}(X) \longrightarrow 0,
$$

where $J_{G}^{r}(X)$ is the $r$-th Griffiths intermediate Jacobian and $H d g^{r}(X)$ is the group of Hodge $r$-cycles generalizes to arbitrary schemes and in more general indices.

Definition 4.1 (A special case of $[$ Car79]). Let $X$ be a scheme over $\mathbb{C}$ and $(m, n) \in$ $\mathbb{Z} \times \mathbb{Z}$. The $(m, n)$-th intermediate Jacobian (resp., with compact supports) of $X$ is defined as

$$
\begin{aligned}
J^{m, n}(X) & :=H_{B}^{m-1}(X, \mathbb{C}) /\left(F^{n} H_{B}^{m-1}(X, \mathbb{C})+H_{B}^{m-1}(X, \mathbb{Z}(n))\right) \\
\left(\text { resp. }, J_{c}^{m, n}(X)\right. & \left.:=H_{c, B}^{m-1}(X, \mathbb{C}) /\left(F^{n} H_{c, B}^{m-1}(X, \mathbb{C})+H_{c, B}^{m-1}(X, \mathbb{Z}(n))\right)\right) .
\end{aligned}
$$

Let us suppose $X$ is connected for the moment. While we have by definition $J_{c}^{2 r, r}(X)=J_{c}^{2 r, r}(X)=J_{G}^{r}(X)$ for smooth proper $X$, an $(m, n)$-th intermediate Jacobian (with compact supports) is not, in general, compact as a complex Lie group. However, by [Car79, Lemma 6], $J_{c}^{m, n}(X)\left(\right.$ resp., $\left.J^{m, n}(X)\right)$ is still a generalized torus, 
i.e. a quotient of a complex vector space by a discrete subgroup, if $m \leqslant 2 n$ (resp., $\min (2 m-1,2 \operatorname{dim} X+1) \leqslant 2 n)$ because the highest possible weight of $H_{c, B}^{m-1}(X, \mathbb{C})$ $\left(\right.$ resp., $\left.H_{B}^{m-1}(X, \mathbb{C})\right)$ is $m-1$ (resp., $\left.\min \{2 m-2,2 \operatorname{dim} X\}\right)$ by [Del74, Théorème 8.2.4].

Proposition 4.2. Let $i: H_{c, B}^{m}(X, \mathbb{Z}(n)) \rightarrow H_{c, B}^{m}(X, \mathbb{C})$ be the map induced by the inclusion $\mathbb{Z}(n) \hookrightarrow \mathbb{C}$. Then, for any scheme $X$ over $\mathbb{C}$, there is a short exact sequence

$$
0 \longrightarrow J_{c}^{m, n}(X) \longrightarrow H_{c, \mathcal{D} B}^{m}(X, \mathbb{Z}(n)) \longrightarrow i^{-1}\left(F^{n} H_{c, B}^{m}(X, \mathbb{C})\right) \longrightarrow 0 .
$$

Also, there is an equality:

$$
i^{-1}\left(F^{n} H_{c, B}^{m}(X, \mathbb{C})\right)=H_{c, B}^{m}(X, \mathbb{Z}(n))_{\text {tor }}
$$

if $m<2 n$.

Analogous statements hold without compact supports except that the analogue of the equality (12) is valid only under the condition $h:=\min (2 m, 2 \operatorname{dim} X)<2 n$.

Proof. It is immediate from Proposition 3.7 and the fact that the maximal weight of $H_{c, B}^{m}(X, \mathbb{C})\left(\right.$ resp., $\left.H_{B}^{m}(X, \mathbb{C})\right)$ is $m$ (resp., $\left.h\right)$.

\section{Deligne-Beilinson cycle maps}

In this section, we construct a cycle map from Suslin-Friedlander's motivic complex $\mathbb{Z}(n)^{S F}$ to the Deligne-Beilinson complex $D B(n)_{\text {Zar }}$. We follow the method of $[\mathbf{B l o 8 6}]$ and [GL01]. Taking the eh sheafification, we obtain Deligne-Beilinson cycle maps for Lichtenbaum cohomology with and without compact supports of arbitrary schemes over $\mathbb{C}$. By Lichtenbaum cohomology (resp., with compact supports) for singular schemes, we mean the eh-hypercohomology (resp., with compact supports) of the Suslin-Friedlander motivic complex.

Recall that Suslin-Friedlander's motivic complex $\mathbb{Z}(n)^{S F}:=C_{\bullet} z_{\text {equi }}\left(\mathbb{A}^{n}, 0\right)[-2 n]$ is a complex of Zariski (even étale) sheaves quasi-isomorphic to Voevodsky's motivic complex on the big Zariski site over any perfect field [MVW06, Theorem 16.7]. We use the fact that $\mathbb{Z}(n)^{S F}$ is a subcomplex of Bloch's cycle complex. More precisely, the inclusion of cycles is compatible with face maps; thus it induces a chain map

$$
i: \mathbb{Z}(n)^{S F}[2 n](X) \hookrightarrow z^{n}\left(X \times \mathbb{A}^{n}, \bullet\right),
$$

for any scheme $X$ [MVW06, Lemma 19.4]. The advantage of the Suslin-Friedlander's complex over Bloch's is that it is defined on a big site while Bloch's does not have enough contravariant functoriality. Hence, the eh-sheafification process applies only to the former.

Now, suppose $X$ is a smooth scheme over $\mathbb{C}$ and $W=\Sigma_{i} n_{i} W_{i}\left(n_{i} \in \mathbb{Z}\right.$ and $W_{i}$ are elementary cycles) is a codimension $n$ cycle on $X$. Choose a smooth compactification $\bar{X}$ and put $\bar{W}:=\Sigma_{i} n_{i} \bar{W}_{i}$, where $\bar{W}_{i}$ is the closure of $W_{i}$ in $\bar{X}$. Let $c_{\mathcal{D}}(\bar{W}) \in$ $H_{\mathcal{D B}, \overline{\operatorname{supp} W}}^{2 n}(\bar{X}, \mathbb{Z}(n))$ be the fundamental class defined in $[\mathbf{E V 8 8}, 7.1]$. The class $c^{\mathcal{D B}}(W) \in H_{\mathcal{D} \mathcal{B}, \operatorname{supp} W}^{2 n}(X, \mathbb{Z}(n))$ is defined as the image of $c_{\mathcal{D}}(\bar{W}) \in H_{\mathcal{D B}, \overline{\operatorname{supp} W}}^{2 n}(\bar{X}, \mathbb{Z}(n))$ under the restriction $H_{\mathcal{D B}, \overline{\operatorname{supp} W}}^{2 n}(\bar{X}, \mathbb{Z}(n)) \rightarrow H_{\mathcal{D} \mathcal{B}, \operatorname{supp} W}^{2 n}(X, \mathbb{Z}(n))$ (see [loc. cit., Remark 7.2]). By the standard argument, we can easily see that the class $c^{\mathcal{D B}}(W)$ does not depend on the compactification $\bar{X}$. 
Lemma 5.1. Let $f: X \rightarrow Y$ be a morphism of smooth schemes, and let $W$ be a codimension $n$ cycle on $Y$ such that the pullback $f^{*} W$ is also a codimension $n$ cycle on $X$. Then, we have $c_{\mathcal{D B}}\left(f^{*} W\right)=f^{*}\left(c_{\mathcal{D B}}(W)\right)$ in $H_{\mathcal{D} \mathcal{B}, \text { supp } f^{*} W}^{2 n}(X, \mathbb{Z}(n))$.

Proof. This follows immediately from the proper case treated in [EV88, Proposition 7.5].

We have the following diagram, in which: (a) $t r_{\leqslant}$means a good truncation of a complex, (b) For a sheaf $\mathcal{F}$ on a scheme $S$ with a closed subscheme $T \hookrightarrow S$, we write $\Gamma_{T}(S, \mathcal{F}):=\operatorname{cone}(\mathcal{F}(S) \rightarrow \mathcal{F}(S \backslash T))[-1]$, and $(\mathrm{c}) D B(n)_{Z a r} \rightarrow \mathcal{K} \bullet$ is an injective resolution in $S h_{Z a r}(S m / \mathbb{C})$.

$$
\begin{aligned}
& C_{r} z_{\text {equi }}\left(\mathbb{A}^{n}, 0\right)(X) \stackrel{c^{\mathcal{D B}}}{\longrightarrow} \operatorname{colim}_{W \in C_{r} z_{\text {equi }}\left(\mathbb{A}^{n}, 0\right)(X)} H_{\mathcal{D} \mathcal{B}, \operatorname{supp} W}^{2 n}\left(X \times \mathbb{A}^{n} \times \Delta^{r}, \mathbb{Z}(n)\right) \\
& \cong \text { Theorem 2.5(i) } \\
& \operatorname{colim}_{W} H_{Z a r, \operatorname{supp} W}^{2 n}\left(X \times \mathbb{A}^{n} \times \Delta^{r}, D B(n)_{Z a r}\right) \\
& \text { qis } \uparrow a \\
& \operatorname{colim}_{W} \operatorname{tr} \leqslant 2 n \Gamma_{\operatorname{supp} W}\left(X \times \mathbb{A}^{n} \times \Delta^{r}, \mathcal{K} \bullet\right)[2 n] \\
& \downarrow \\
& \Gamma\left(X \times \mathbb{A}^{n} \times \Delta^{r}, \mathcal{K}^{\bullet}\right)[2 n],
\end{aligned}
$$

where the map $a$ is a quasi-isomorphism by the weak purity of Deligne-Beilinson cohomology, and $b$ is the map that forgets the truncation and supports.

Since we are only dealing with equidimensional cycles, all maps in the diagram (13) are compatible with pullbacks along face maps and contravariant in $X$ with respect to all morphisms by Lemma 5.1. Therefore, we obtain the maps of complexes of presheaves on $S m / \mathbb{C}$ :

$$
\begin{aligned}
& \mathbb{Z}(n)^{S F}[2 n] \stackrel{\text { def. }}{=} C_{\bullet} z_{\text {equi }}\left(\mathbb{A}^{n}, 0\right)(-) \\
& \underbrace{c^{\mathcal{D B}}} \\
& \operatorname{colim}_{W \in C}{\text { z } z_{\text {equi }}\left(\mathbb{A}^{n}, 0\right)(-)}^{\longrightarrow} H_{Z a r, \operatorname{supp} W}^{2 n}\left(-\times \mathbb{A}^{n} \times \Delta^{\bullet}, D B(n)_{Z a r}\right) \\
& \text { qis } \uparrow^{\uparrow} \\
& \operatorname{Tot}^{\oplus} \operatorname{colim}_{W} \operatorname{tr} \leqslant 2 n \Gamma_{\operatorname{supp} W}\left(-\times \mathbb{A}^{n} \times \Delta^{\bullet}, \mathcal{K} \bullet\right)[2 n] \\
& b \\
& \operatorname{Tot}^{\oplus} \Gamma\left(-\times \mathbb{A}^{n} \times \Delta^{\bullet}, \mathcal{K}^{\bullet}\right)[2 n] .
\end{aligned}
$$

Furthermore, the homotopy invariance of the Deligne-Beilinson cohomology implies that the following two maps are quasi-isomorphisms:

$$
\operatorname{Tot}^{r, s}:=\operatorname{Tot}_{r, s}^{\oplus} \Gamma\left(U \times \mathbb{A}^{n} \times \Delta^{r}, \mathcal{K}^{s}\right) \stackrel{\text { qis }}{\longleftarrow} \Gamma\left(U \times \mathbb{A}^{n}, \mathcal{K}^{\bullet}\right) \stackrel{\text { qis }}{\longleftarrow} \Gamma(U, \mathcal{K} \bullet),
$$


where the first arrow is the inclusion of $\Gamma\left(U \times \mathbb{A}^{n}, \mathcal{K}^{s}\right)$ to the $(0, s)$-th direct summand $\Gamma\left(U \times \mathbb{A}^{n} \times \Delta^{0}, \mathcal{K}^{s}\right)$ of the total complex and the second is induced by the projection $U \times \mathbb{A}^{n} \rightarrow U$. Since these two quasi-isomorphisms are contravariant in $U$, they give maps of complexes of Zariski sheaves on $\mathrm{Sm} / \mathbb{C}$.

Combining the diagrams (14) and (15), we obtain the maps of presheaves on $\mathrm{Sm} / \mathbb{C}$

$$
\begin{aligned}
& \mathbb{Z}(n)^{S F} \stackrel{c^{\mathcal{D B}}}{\longrightarrow} \operatorname{colim}_{W \in C \bullet z_{\text {equi }}\left(\mathbb{A}^{n}, 0\right)(X)} H_{Z a r, \operatorname{supp} W}^{2 n}\left(-\times \mathbb{A}^{n} \times \Delta^{\bullet}, D B(n)_{Z a r}\right)[-2 n] \\
& \text { qis } \uparrow a \\
& \operatorname{Tot}^{\oplus} \operatorname{colim}_{W} t_{\leqslant 2 n} \Gamma_{\text {supp } W}\left(-\times \mathbb{A}^{n} \times \Delta^{\bullet}, \mathcal{K}^{\bullet}\right) \\
& \downarrow b \\
& \operatorname{Tot}^{\oplus} \Gamma\left(-\times \mathbb{A}^{n} \times \Delta^{\bullet}, \mathcal{K}^{\bullet}\right) \\
& \text { qis } \uparrow_{\mathcal{K}}^{\bullet} \\
& \text { qis as Zariski sheaves } \\
& D B(n)_{Z a r} .
\end{aligned}
$$

Taking the Zariski sheafification, we obtain the corresponding diagram of complexes of Zariski sheaves on $S m / \mathbb{C}$. Let us record this as a theorem.

Theorem 5.2. There is a map

$$
c^{\mathcal{D B}}(n): \mathbb{Z}(n)^{S F} \longrightarrow D B(n)_{Z a r}
$$

in $D\left(S h_{\text {Zar }}(S m / \mathbb{C})\right)$ such that the induced map of Zariski hypercohomology of any smooth scheme $X$

$$
H_{Z a r}^{m}\left(X, \mathbb{Z}(n)^{S F}\right) \longrightarrow H_{Z a r}^{m}\left(X, D B(n)_{Z a r}\right) \cong H_{\mathcal{D B}}^{m}(X, \mathbb{Z}(n))
$$

agrees with the Deligne-Beilinson cycle map in [Blo86] via the canonical isomorphism $H_{Z a r}^{m}\left(X, \mathbb{Z}(n)^{S F}\right) \cong C H^{n}(X, 2 n-m)$ constructed in [MVW06, Chapter 19].

Proof. This follows from the construction of $c^{\mathcal{D B}}(n)$ and [MVW06, Theorem 19.8, Proposition 19.12].

By eh sheafification, $c^{\mathcal{D B}}(n)$ induces the map

$$
c_{e h}^{\mathcal{D B}}(n): \mathbb{Z}(n)_{e h}^{S F} \longrightarrow D B(n)_{e h}^{\mathcal{D B}}
$$

in $D\left(S h_{e h}(S c h / \mathbb{C})\right)$.

Definition 5.3. Let $X$ be an arbitrary scheme over $\mathbb{C}$. The Deligne-Beilinson cycle map for Lichtenbaum cohomology (resp., with compact supports)

$$
\begin{aligned}
c l_{L}^{\mathcal{D} \mathcal{B}}: H_{L}^{m}(X, \mathbb{Z}(n)) & \longrightarrow H_{\mathcal{D B}}^{m}(X, \mathbb{Z}(n)) \\
\left(\operatorname{resp}_{,}, c l_{c, L}^{\mathcal{D B}}: H_{c, L}^{m}(X, \mathbb{Z}(n))\right. & \left.\longrightarrow H_{c, \mathcal{D} \mathcal{B}}^{m}(X, \mathbb{Z}(n))\right)
\end{aligned}
$$

is the map induced by taking the eh hypercohomology (resp., with compact supports) of $c_{e h}^{\mathcal{D B}}(n)$. 
The same construction applied to the Betti cycle class - note that Betti cohomology also has weak purity and $\mathbb{A}^{1}$-homotopy invariance-gives the morphism

$$
c^{B}(n): \mathbb{Z}(n)^{S F} \longrightarrow B(n)_{Z a r}
$$

in $D\left(S h_{Z a r}(S m / \mathbb{C})\right)$. Taking the eh hypercohomology, we define Betti cycle maps for Lichtenbaum cohomology (resp., with compact supports)

$$
c l_{(c), L}^{B}: H_{(c), L}^{m}(X, \mathbb{Z}(n)) \longrightarrow H_{(c), B}^{m}(X, \mathbb{Z}(n)),
$$

for an arbitrary $X \in S c h / \mathbb{C}$.

By construction and the definition of the Deligne-Beilinson cycle map (it is defined by lifting Betti fundamental classes; see $[\mathbf{E V 8 8},(7.1)])$, there is a commutative diagram

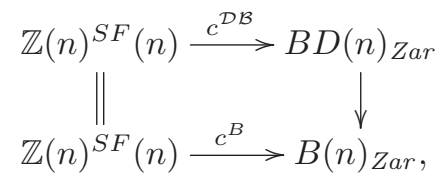

where the right vertical arrow is the map induced by the projection $\left\{D B(n)_{\bar{T}, T}\right\} \rightarrow$ $\left\{B(n)_{\bar{T}, T}\right\}$ in $S h_{a n}(\Pi)$.

With Proposition 4.2 and the eh sheafification of the diagram (16), the DeligneBeilinson cycle maps restrict to the homological part of $H_{(c), L}^{m}(X, \mathbb{Z}(m))$ (resp., $\left.H_{c, L}^{m}(X, \mathbb{Z}(m))\right)$

$$
H_{(c), L, h o m}^{m}(X, \mathbb{Z}(m)):=\operatorname{ker}\left\{H_{(c), L}^{m}(X, \mathbb{Z}(m)) \stackrel{c l_{(c), L}^{B}}{\longrightarrow} H_{(c), B}^{m}(X, \mathbb{Z}(n))\right\}
$$

as indicated in the following diagram:

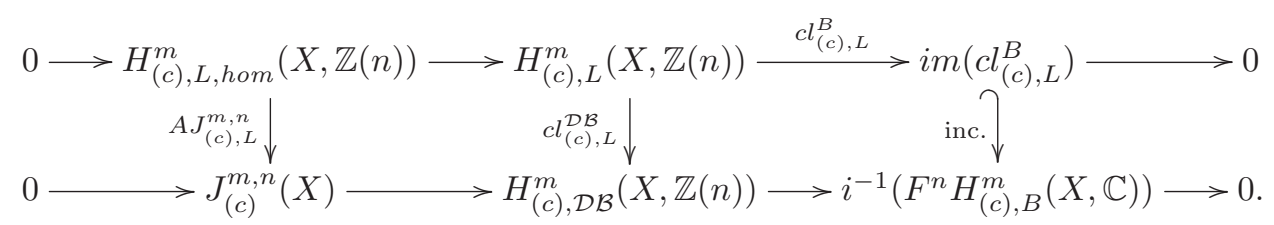

Here, $A J_{(c), L}^{m, n}$ is defined as the restriction of $c l_{(c), L}^{\mathcal{D B}}$ and $i: H_{(c), B}^{m}(X, \mathbb{Z}(n)) \rightarrow$ $H_{(c), B}^{m}(X, \mathbb{C})$ is the map induced by the inclusion $\mathbb{Z}(n) \hookrightarrow \mathbb{C}$. Let us point out that $H_{(c), L, h o m}^{m}(X, \mathbb{Z}(m))$ coincides with the subgroup of divisible elements of $H_{(c), L}^{m}(X$, $\mathbb{Z}(m))$ as explained in Remark 6.2 below.

Theorem 5.4 (Abel-Jacobi and Lefschetz theorems; cf. [Ara16, Theorem 7.8]). For an arbitrary $X \in S c h / \mathbb{C}$,

i. The change of topologies induces an isomorphism $H_{c, c d h}^{2}\left(X, \mathbb{Z}(1)_{c d h}^{S F}\right) \rightarrow H_{c, L}^{2}(X$, $\mathbb{Z}(1))$.

ii. The Abel-Jacobi map with compact supports

$$
A J_{c}^{2,1}: H_{c, L, h o m}^{2}(X, \mathbb{Z}(1)) \longrightarrow J_{c}^{2,1}(X)
$$

is an isomorphism. 
iii. The Betti cycle map with compact supports

$$
c l_{c}^{B}: H_{c, L}^{2}(X, \mathbb{Z}(1)) \longrightarrow H_{c, B}^{2}(X, \mathbb{Z}(1))
$$

is surjective onto $i^{-1}\left(F^{1} H_{c, B}^{2}(X, \mathbb{C})\right)$.

Proof. For $i$, since $\mathbb{Z}(1)^{S F}$ is quasi-isomorphic to $\mathbb{G}_{m}[-1]$ on $S m / \mathbb{C}_{Z a r}$, it suffices to show that the canonical map $H_{c, c d h}^{1}\left(X, \mathbb{G}_{m, c d h}\right) \rightarrow H_{c, e h}^{1}\left(X, \mathbb{G}_{m, e h}\right)$ is an isomorphism. Since both cdh and eh cohomologies with compact supports have localization sequences, it suffices to prove that the change of topologies induces isomorphisms $H_{c d h}^{i}\left(S, \mathbb{G}_{m, c d h}\right) \cong H_{e h}^{i}\left(S, \mathbb{G}_{m, e h}\right)$ for $i=0$ and 1 for arbitrary proper schemes $S$ over $\mathbb{C}$. Considering the spectral sequences for both sides associated with a smooth cdh (a fortiori eh) hypercover of $S$, we may further assume that $S$ is smooth. The desired isomorphisms follow from [MVW06, Proposition 13.27] (or Theorem 3.1 for the étale case) and Hilbert's Theorem 90 [Mil80, Chapter III, Proposition 4.9].

For $i$ and $i i$, by the diagram (17), it suffices to show that the map

$$
c l_{c, L}^{\mathcal{D B}}: H_{c, L}^{2}(X, \mathbb{Z}(1)) \longrightarrow H_{c, \mathcal{D B}}^{2}(X, \mathbb{Z}(1))
$$

is an isomorphism. The source and the target are both defined by eh hypercohomology, and the cycle map is by definition induced by the morphism $c_{e h}^{\mathcal{D B}}(1): \mathbb{Z}(1)_{e h}^{S F} \rightarrow$ $D B(1)_{e h}^{\mathcal{D} \mathcal{B}}$ of complexes of eh sheaves in the derived category. Thus, taking a compactification of $X$ and arguing with localization sequences and the 5-lemma, we can see that it suffices to prove that the cycle map

$$
c l_{L}^{\mathcal{D} \mathcal{B}}: H_{L}^{i}(S, \mathbb{Z}(1)) \longrightarrow H_{\mathcal{D B}}^{i}(S, \mathbb{Z}(1))
$$

is an isomorphism for any proper scheme $S$ over $\mathbb{C}$ if $i=1$ or 2 .

Let $S_{\bullet} \rightarrow S$ be a smooth proper cdh hypercover of $S$ and consider the maps of spectral sequences

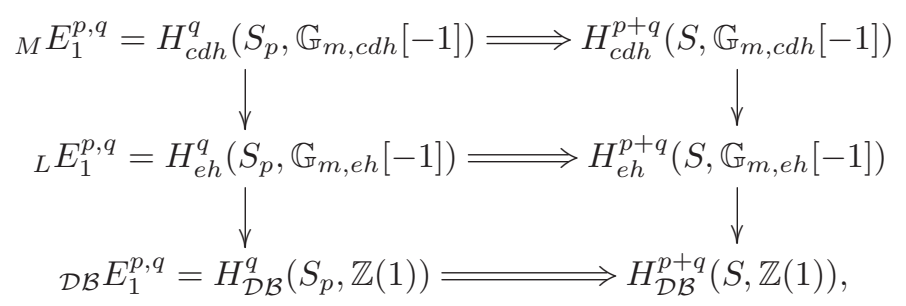

where the top vertical maps are induced by the change of topologies (same as in $i$ except for the indexing) and the bottom ones by the composition of $c_{e h}^{\mathcal{D B}}(1): \mathbb{Z}(1)_{e h}^{S F}$ $\rightarrow D B(1)_{e h}^{\mathcal{D B}}$ with the canonical quasi-isomorphism $\mathbb{G}_{m, e h}[-1] \simeq \mathbb{Z}(1)_{e h}^{S F}$.

The compositions ${ }_{M} E_{1}^{p, q} \rightarrow{ }_{L} E_{1}^{p, q} \rightarrow{ }_{\mathcal{D B}} E_{1}^{p, q}$ of the $E_{1}$-terms are isomorphisms for $q \leqslant 2$. Indeed, it is trivial if $q=0$. For $q=1$ or 2 , since $S_{p}$ is smooth and proper, the claim is equivalent to that Bloch's cycle maps $C H^{1}\left(S_{p}, 2-q\right) \rightarrow H_{\mathcal{D B}}^{q}\left(S_{p}, \mathbb{Z}(1)\right)$ are isomorphisms for $q=1$ and 2. If $q=1$, we may assume that $S_{p}=$ Spec $\mathbb{C}$ because the structure morphism of any smooth proper connected scheme induces isomorphisms of both higher Chow group $C H^{1}(-, 1)$ and Deligne-Beilinson cohomology $H_{\mathcal{D} \mathcal{B}}^{1}(-, \mathbb{Z}(1))$. In this case, the cycle map is indeed an isomorphism $C H^{1}(\operatorname{Spec} \mathbb{C}, 1) \cong$ 
$\mathbb{C}^{*} \stackrel{\log }{\longrightarrow} \mathbb{C} / 2 \pi i \mathbb{Z} \cong H_{\mathcal{D} \mathcal{B}}^{1}$ (Spec $\left.\mathbb{C}, \mathbb{Z}(1)\right)$ by [KLM-S06, Section 5.7]. The case for $q=2$ follows from the Abel-Jacobi theorem and the Lefschetz theorem for smooth proper schemes by the diagram (1).

Now, as we have seen in the proof for $i$, the first map ${ }_{M} E_{1}^{p, q} \rightarrow{ }_{L} E_{1}^{p, q}$ is an isomorphism if $q \leqslant 2$, so the second map ${ }_{L} E_{1}^{p, q} \rightarrow \mathcal{D B B}_{1}^{p, q}$ is also an isomorphism if $q \leqslant 2$. Therefore, we conclude that

$$
c l_{L}^{\mathcal{D B}}: H_{L}^{i}(S, \mathbb{Z}(1)) \cong H_{e h}^{i}\left(S, \mathbb{G}_{m, e h}[-1]\right) \longrightarrow H_{\mathcal{D B}}^{i}(S, \mathbb{Z}(1))
$$

is an isomorphism for any proper $S$ if $i=1$ or 2 .

\section{Torsion part of cycle maps}

By the commutativity of the diagram (16), we have a map of distinguished triangles for any positive integer $a$

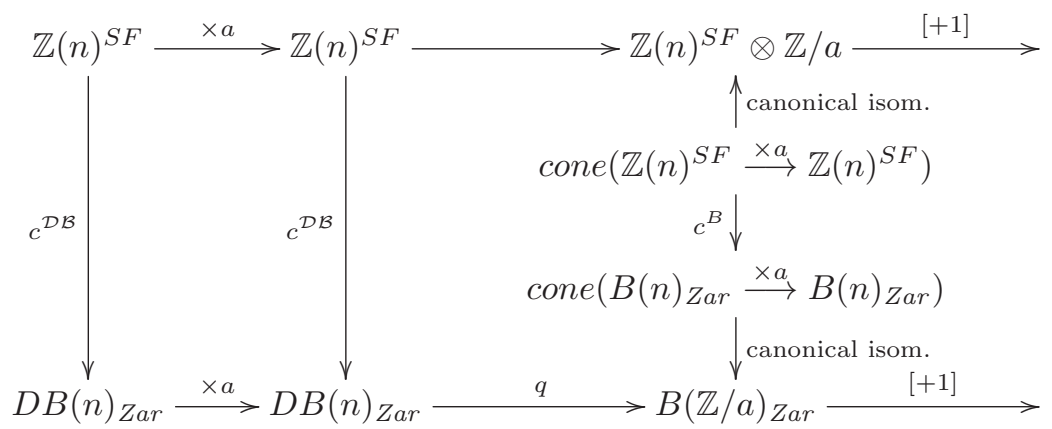

with the bottom triangle being the one in (8).

Lemma 6.1. The étale sheafification of the composition of the far right vertical arrows in the diagram (18)

$$
F: \mathbb{Z}(n)^{S F} \otimes \mathbb{Z} / a \longrightarrow B(\mathbb{Z} / a)_{\text {ét }}
$$

is an isomorphism in $D\left(S h_{e ́ t}(S m / \mathbb{C})\right)$.

Proof. Let $\mathcal{I}^{\bullet}$ be an injective resolution of $\mathbb{Z} / a$ in the cl-topology and let $o: S h_{c l}$ $(M f d / \mathbb{C}) \rightarrow S h_{e ́ t}(S m / \mathbb{C})$ be the restriction along the complex analytification functor $h: S m / \mathbb{C} \rightarrow M f d / \mathbb{C}$. Then, there is a natural comparison map by Artin between $\mathbb{Z} / a$ coefficient Betti cohomology and $\mu_{a}^{\otimes n}$-coefficient étale cohomology given by a quasiisomorphism of complexes of étale sheaves $A: \mu_{a}^{\otimes n} \stackrel{\text { qis }}{\longrightarrow} R o_{*}\left(\mathcal{I}^{\bullet}\right)$; see [Mil80, Chapter III, Lemma 3.15] and the paragraph that precedes it.

As we have explained in the second paragraph of the proof of Theorem $2.5, R o_{*}\left(\mathcal{I}^{\bullet}\right)$ is precisely our $B(\mathbb{Z} / a)_{e ́ t}$. By the same construction of a cycle map as in Section 5 (but this time with étale hypercohomology instead of Zariski hypercohomology) we may lift the étale cycle map to the map $c^{e ́ t}: \mathbb{Z}(n)^{S F} \rightarrow \mu_{a}^{\otimes n}$ in $D\left(S h_{e ́ t}(S m / \mathbb{C})\right)$. Since Betti and étale cycle maps are compatible with Artin's comparison map, we have a 
commutative diagram

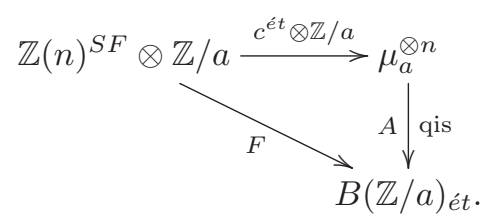

Now, $c^{e ́ t} \otimes \mathbb{Z} / a: \mathbb{Z}(n)^{S F} \otimes \mathbb{Z} / a \rightarrow \mu_{a}^{\otimes n}$ is a quasi-isomorphism by [GL01, Theorem 1.5]. Therefore $F$ is also a quasi-isomorphism.

Remark 6.2 (Interlude; cf. [Gei17, Section 3]). For any scheme $X$ over $\mathbb{C}$, the homological part $H_{c, L, h o m}^{m}(X, \mathbb{Z}(n))$ (resp., $H_{L, h o m}^{m}(X, \mathbb{Z}(n))$ ) is the subgroup of all divisible elements of $H_{c, L}^{m}(X, \mathbb{Z}(n))$ (resp., $H_{L}^{m}(X, \mathbb{Z}(n))$ ) as can be seen from the following commutative diagram of cohomology groups (resp., with compact supports):

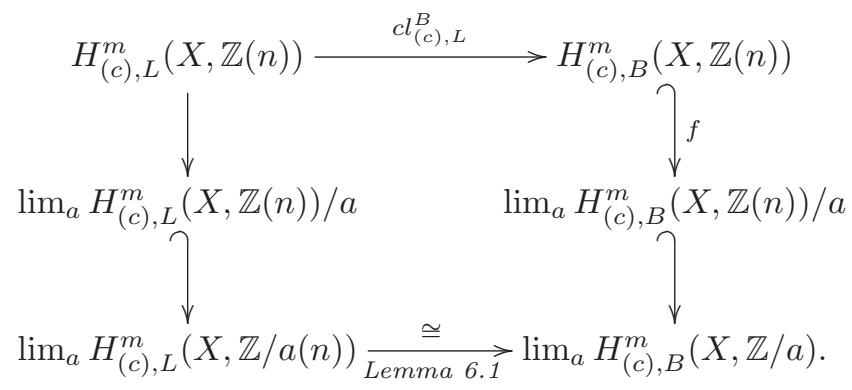

Here, $f$ is injective because $H_{(c), B}^{m}(X, \mathbb{Z}(n))$ is finitely generated.

Proposition 6.3. For any $X \in S c h / \mathbb{C}$, there is a commutative diagram

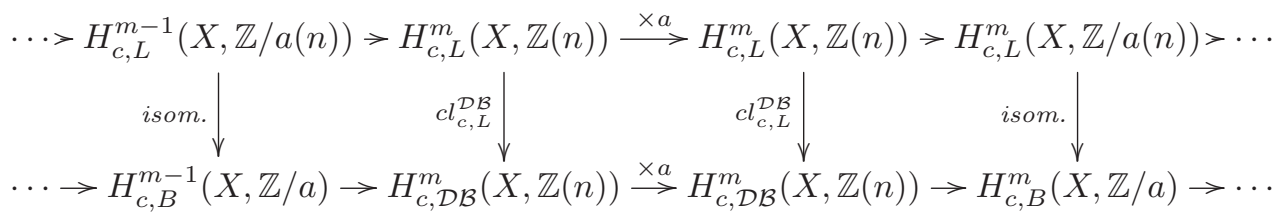

such that the horizontal rows are exact. An analogous statement for cohomology without compact supports also holds.

Proof. Take the eh sheafification of the diagram (18) and pass to the eh hypercohomology. The proposition follows from Corollary 3.2, Definition 3.5 and Lemma 6.1.

Theorem 6.4 (cf. [RS16, Proposition 5.1]). Let $X$ be an arbitrary scheme over $\mathbb{C}$ and let $n$ be a positive integer. Then,

i. $c_{c, L}^{\mathcal{D B}}: H_{c, L}^{m}(X, \mathbb{Z}(n)) \rightarrow H_{c, \mathcal{D} B}^{m}(X, \mathbb{Z}(n))$ is surjective on torsion and has a torsion-free cokernel. Also, so is $\operatorname{cl}_{L}^{\mathcal{D B}}: H_{L}^{m}(X, \mathbb{Z}(n)) \rightarrow H_{\mathcal{D B}}^{m}(X, \mathbb{Z}(n))$.

ii. Let $m \leqslant 2 n$. Then, $c l_{c, L}^{\mathcal{D B}}: H_{c, L}^{m}(X, \mathbb{Z}(n)) \rightarrow H_{c, \mathcal{D} \mathcal{B}}^{m}(X, \mathbb{Z}(n))$ is an isomorphism on torsion, $H_{c, \mathcal{D} B}^{m-1}(X, \mathbb{Z}(n)) \otimes \mathbb{Q} / \mathbb{Z}=0$, and $H_{c, \mathcal{D B}}^{m}(X, \mathbb{Z}(n))_{\text {tor }} \cong H_{c, B}^{m-1}(X, \mathbb{Q} / \mathbb{Z})$.

iii. Let $\min \{2 m-1,2 \operatorname{dim} X+1\} \leqslant 2 n$. Then, $c l_{L}^{\mathcal{D} B}: H_{L}^{m}(X, \mathbb{Z}(n)) \rightarrow H_{\mathcal{D} B}^{m}(X, \mathbb{Z}(n))$ is an isomorphism on torsion, $H_{\mathcal{D B}}^{m-1}(X, \mathbb{Z}(n)) \otimes \mathbb{Q} / \mathbb{Z}=0$, and $H_{\mathcal{D} B}^{m}(X, \mathbb{Z}(n))_{\text {tor }}$ $\cong H_{B}^{m-1}(X, \mathbb{Q} / \mathbb{Z})$. 
Proof. With Proposition 6.3, the proof of [RS16, Proposition 5.1] applies.

Corollary 6.5. In the diagram (17) for any $X \in S c h / \mathbb{C}$,

i. $A J_{c}^{m, n}: H_{c, L, h o m}^{m}(X, \mathbb{Z}(n)) \rightarrow J_{c}^{m, n}(X) \quad$ (resp., $\quad A J^{m, n}: H_{L, h o m}^{m}(X, \mathbb{Z}(n)) \rightarrow$ $J^{m, n}(X)$ ) is an isomorphism on torsion if $m \leqslant 2 n$ (resp., $\min \{2 m-1,2 \operatorname{dim} X$ $+1\} \leqslant 2 n)$. In these ranges, if $X$ is connected, the Jacobians $J_{(c)}^{m, n}(X)$ are generalized complex tori.

ii. The quotient group $i^{-1}\left(F^{n} H_{(c), B}^{m}(X, \mathbb{C})\right) / i m\left(c l_{(c), L}^{B}\right)$ is always torsion free. In particular,

$$
\begin{array}{r}
c l_{c, L}^{B}: H_{c, L}^{m}(X, \mathbb{Z}(n)) \longrightarrow H_{c, B}^{m}(X, \mathbb{Z}(n)) \\
\left(\text { resp., cl } l_{L}^{B}: H_{L}^{m}(X, \mathbb{Z}(n)) \longrightarrow H_{B}^{m}(X, \mathbb{Z}(n))\right)
\end{array}
$$

has the image

$$
\begin{aligned}
i^{-1}\left(F^{n} H_{c, B}^{m}(X, \mathbb{C})\right) & =H_{c, B}^{m}(X, \mathbb{Z}(n))_{\text {tor }} \\
\left(\text { resp. }, i^{-1}\left(F^{n} H_{B}^{m}(X, \mathbb{C})\right)\right. & \left.=H_{B}^{m}(X, \mathbb{Z}(n))_{\text {tor }}\right)
\end{aligned}
$$

if $m<2 n$ (resp., $\min \{2 m, 2 \operatorname{dim} X\}<2 n$ ).

Proof. The first part of $i$ is immediate from Theorem 6.4 (ii) and (iii). The second part of $i$ is already explained in the paragraph right after Definition 4.1.

For $i$, taking the cokernels of the vertical arrows in the diagram (17), we obtain the exact sequence

$$
0 \longrightarrow \operatorname{coker} A J_{(c)}^{m, n} \longrightarrow \operatorname{coker} c l_{(c), L}^{\mathcal{D B}} \longrightarrow i^{-1}\left(F^{n} H_{(c), B}^{m}(X, \mathbb{C})\right) / i m\left(c l_{(c), L}^{B}\right) \longrightarrow 0 .
$$

The torsion-freeness of $i^{-1}\left(F^{n} H_{(c), B}^{m}(X, \mathbb{C})\right) / i m\left(c l_{(c), L}^{B}\right)$ follows because coker $A J_{(c)}^{m, n}$, being a quotient of a vector space, is divisible and coker $c l_{(c), L}^{\mathcal{D B}}$ is torsion-free by Theorem $6.4(i)$. The final assertions are now clear with the equalities (12).

Remark 6.6. Let $X$ be a projective scheme of dimension d. Krishna and Pelaez [KP17, Theorem 1.5] proved a result similar to Corollary 6.5 (i) and more for the zero cycle case (i.e. when $m=2 n=2 d$ ). Their theorem is about Voevodsky's motivic cohomology $H^{2 d}(X, \mathbb{Z}(d)$ ) (not about Lichtenbaum cohomology) and they constructed the Abel-Jacobi maps via Chern class homomorphisms from Weibel's homotopy invariant $K$-theory.

They proved the surjectivity and the torsion isomorphism property of the AbelJacobi maps for projective schemes of dimension at most 2 or of any dimension but regular in codimension 1. Up to the question whether our and their Abel-Jacobi maps agree, for schemes of dimension at most 2 , our and their results on the torsion part can be translated into each other because the torsion subgroups of $H^{2 d}(X, \mathbb{Z}(d))$ and $H_{L}^{2 d}(X, \mathbb{Z}(d))$ are canonically isomorphic by the Rost-Voevodsky norm residue isomorphism theorem. For higher dimensional schemes $(d \geqslant 3)$, we are out of the range of the norm residue isomorphism theorem, so another approach is required. Krishna and Pelaez's proof uses the Rojtman torsion theorem for Levine-Weibel Chow groups due to Biswas and Srinivas [BS99]. Lastly, in our article, we are not dealing with the surjectivity assertion. 


\section{Griffiths intermediate Jacobians revisited}

With Theorem 6.4, we may characterize the "algebraic part" of Griffiths intermediate Jacobians by a universal property. In the rest of this paper, we assume that $X$ is smooth, proper and connected.

Samuel [Sam58] introduced regular homomorphisms to relate the algebraic part $C H_{\text {alg }}^{r}(X):=\left\{z \in C H^{r}(X) \mid z\right.$ is algebraically equivalent to 0$\}$ of the Chow group $C H^{r}(X)$ of $X$ over an arbitrary algebraically closed field $k$ with abelian varieties over the same base field $k$. It is known that, with no further restriction on the base field, there is a universal regular homomorphism if the codimension $r$ is 1 or $\operatorname{dim} X$ [ibid.], but the existence is unknown in other codimensions. However, over the field of complex numbers, we have Abel-Jacobi maps $A J_{X}^{r}: C H_{h o m}^{r}(X) \rightarrow J_{G}^{r}(X)$ for all $r$. Their restrictions to the algebraic part $A J_{X, \text { alg }}^{r}: C H_{\text {alg }}^{r}(X) \rightarrow J_{G}^{r}(X)_{a l g}:=$ $A J_{X}^{r}\left(C H_{\text {alg }}^{r}(X)\right)$ are known to be regular (see [Lib72]) and agree with the universal regular homomorphisms if $r=1,2$ or $\operatorname{dim} X$ [Mur85]. For general $r$, such a statement is not known.

While the existence of universal regular homomorphisms is not known in general, Geisser [Gei17, Section 3] constructed an analogue for Lichtenbaum cohomology $H_{L}^{2 r}(X, \mathbb{Z}(r))$ for any $r$ over any algebraically closed base field $k$. Here, we consider a variant of it.

Consider the canonical natural maps

$$
C H^{r}(X) \cong H^{2 r}(X, \mathbb{Z}(r)) \stackrel{\text { def. }}{=} H_{N i s}^{2 r}\left(X, \mathbb{Z}(r)^{S F}\right) \longrightarrow H_{e t}^{2 r}\left(X, \mathbb{Z}(r)^{S F}\right) \stackrel{\text { def. }}{=} H_{L}^{2 r}(X, \mathbb{Z}(r)),
$$

and define $H_{L, a l g}^{2 r}(X, \mathbb{Z}(r))$ as the image of $C H_{a l g}^{r}(X)$ under (19), contrary to the definition in loc. cit. Let us say that a group homomorphism

$$
\phi: H_{L, a l g}^{2 r}(X, \mathbb{Z}(r)) \longrightarrow A(k),
$$

with $A$ an abelian variety over $k$ is $L$-regular if its composition with the canonical map $C H_{\text {alg }}^{r}(X) \rightarrow H_{L, a l g}^{2 r}(X, \mathbb{Z}(r))$ is regular in Samuel's sense, i.e. for any smooth proper connected $T$ over $k$ and any $Y \in C H^{r}(T \times X)$, the composition

$$
T(k) \stackrel{w_{Y}}{\longrightarrow} C H_{\text {alg }}^{r}(X) \longrightarrow H_{L, \text { alg }}^{2 r}(X, \mathbb{Z}(r)) \stackrel{\phi}{\longrightarrow} A(k)
$$

is a scheme morphism, where $w_{Y}$ sends $t \in T(k)$ to the pullback of $Y$ along $X \cong$ Spec $k \times X \stackrel{t \times i d_{X}}{\longrightarrow} T \times X$.

The existence of the universal $L$-regular homomorphism $\Phi_{L, X}^{r}: H_{L, \text { alg }}^{2 r}(X, \mathbb{Z}(r)) \rightarrow$ $A l g_{L, X}^{r}(k)$ for any $r$ and any smooth proper connected scheme $X$ over $k$ follows by the same argument as in [ibid., Theorem 3.5]. The map $\Phi_{L, X}^{r}$ is surjective and surjective on torsion by the construction. We have the following corollary to Theorem 6.4, which may be regarded as an algebraic construction of the algebraic part of Griffiths intermediate Jacobians and Abel-Jacobi maps.

Corollary 7.1. Let $X$ be a smooth proper connected scheme over $\mathbb{C}$. The composition

$$
C H_{a l g}^{r}(X) \longrightarrow H_{L, a l g}^{2 r}(X, \mathbb{Z}(r)) \stackrel{\Phi_{L, X}^{r}}{\longrightarrow} A l g_{L, X}^{r}(\mathbb{C})
$$

of the universal L-regular homomorphism $\Phi_{L, X}^{r}$ with the canonical map $C H_{a l g}^{r}(X) \rightarrow$ 
$H_{L, a l g}^{2 r}(X, \mathbb{Z}(r))$ is nothing but the algebraic part of the Abel-Jacobi map $A J_{X, a l g}^{r}$ : $C H_{a l g}^{r}(X) \rightarrow J_{G}^{r}(X)_{a l g}$.

Proof. By Theorems $2.5(i)$ and 5.2, the Deligne-Beilinson cycle map factors through Lichtenbaum cohomology as

$$
C H^{r}(X) \longrightarrow H_{L}^{2 r}(X, \mathbb{Z}(r)) \stackrel{c l_{L}^{\mathcal{D} B}}{\longrightarrow} H_{\mathcal{D} \mathcal{B}}^{2 r}(X, \mathbb{Z}(r)) .
$$

Therefore, by [EV88, Theorem 7.11], we obtain the factorization of $A J_{X, \text { alg }}^{r}$

$$
C H_{\text {alg }}^{r}(X) \longrightarrow H_{L, a l g}^{2 r}(X, \mathbb{Z}(r)) \stackrel{A J^{2 r, r}}{\longrightarrow} J_{G}^{r}(X)_{a l g}
$$

where $A J^{2 r, r}$ denotes the restriction of $c l_{L}^{\mathcal{D} \mathcal{B}}$.

Now, we have the diagram

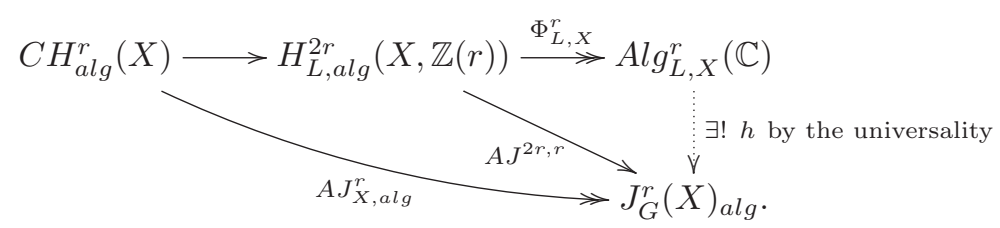

The map $h$ is surjective by the commutativity and also injective on torsion because $A J^{2 r, r}$ is injective on torsion by Theorem $6.4(i i)$ and $\Phi_{L, X}^{r}$ is surjective on torsion by the construction. Since $h$ is induced by a morphism of abelian varieties, it must be an isomorphism.

\section{References}

[Ara16] Arapura, D., A Lefschetz $(1,1)$ theorem for singular varieties. Preprint. Available at: https://arxiv.org/abs/1605.00587.

[Art73] Artin, M., Comparaison avec la cohomologie classique: cas d'un schema lisse, in: Théorie des topos et cohomologie étale des schemas, Tome 3, Lecture Notes in Math. 305, Springer-Verlag, Berlin, 1973, 64-78.

[AH62] Atiyah, M.F., Hirzebruch, F., Analytic cycles on complex manifolds. Topology 1 (1962), 25-45.

[Bei84] Beilinson, A.A., Higher regulators and values of $L$-functions. Sovrem. Probl. Mat. 24, 181-238, Itogi Nauki i Tekhniki, Akad. Nauk SSSR, Vsesoyuz. Inst. Nauchn. i Tekhn. Inform., Moscow, 1984.

[BS99] Biswas, J., Srinivas, V., Roitman's theorem for singular projective varieties. Compos. Math., 119 (1999), 213-237.

[Blo86] Bloch, S., Algebraic cycles and the Beilinson conjectures, in: The Lefschetz centennial conference, Part I (Mexico City, 1984), Contemp. Math., 58, American Mathematical Society, Providence, RI, 1986, 6579.

[Car79] Carlson, J.A., Extensions of mixed Hodge structures. Journées de geométrie algébrique d'Angers, 1979, 107-127. 
[CD12] Cisinski, D.-C., Déglise, F., Triangulated categories of mixed motives. Preprint. Available at: https://arxiv.org/abs/0912.2110.

[Con] Conrad, B., Cohomological descent. Expository note available at: http://math.stanford.edu/ conrad/papers/hypercover.pdf.

[Del71] Deligne, P., Théorie de Hodge: II. Publ. Math. Inst. Hautes Études Sci. 40 (1971), 5-58.

[Del74] Deligne, P., Théorie de Hodge: III. Publ. Math. Inst. Hautes Études Sci. 44 (1974), 5-77.

[EV88] Esnault, H., Viehweg, E., Deligne-Beilinson cohomology, in: Beilinson's conjectures on special values of L-functions, Perspect. Math. 4, Academic Press, Boston, MA, 1988, 43-91.

[FV00] Friedlander, E., Voevodsky, V., Bivariant cycle cohomology, in: Cycles, transfers, and motivic homology theories, Ann. of Math. Stud. 143, Princeton University Press, Princeton, NJ, 2000, 138-187.

[Gei06] Geisser, T., Arithmetic cohomology over finite fields and special values of $\zeta$-functions. Duke Math. J. 133 (2006), no. 1, 27-57.

[Gei17] Geisser, T., On the structure of étale motivic cohomology. J. Pure Appl. Algebra 221 (2017), 1614-1628.

[GL01] Geisser, T., Levine, M., The Bloch-Kato conjecture and a theorem of Suslin-Voevodsky. J. Reine Angew. Math. 530 (2001), 55-103.

[HS15] Holmstrom, A., Scholbach, J., Arakelov motivic cohomology I. J. Algebraic Geom. 24 (2015), 719-754.

[HM-S17] Huber, A., Müller-Stach, S., Periods and Nori motives, Ergeb. Math. Grenzgeb. (3) 65, Springer, 2017.

[KLM-S06] Kerr, M., Lewis, J.D., Müller-Stach, S., The Abel-Jacobi map for higher Chow groups. Compos. Math. 142 (2006), 374-396.

[KP17] Krishna, A., Pelaez, P., The slice spectral sequence for singular schemes and applications. Preprint. Available at: https:// arxiv.org/abs/1606.05810.

[Lib72] Lieberman, D., Intermediate Jacobians, in: Algebraic geometry, Oslo 1970 (Proc. Fifth Nordic Summer-School in Math.), Wolters-Noordhoff, Groningen, 1972, 125-139.

[Mac98] Mac Lane, S., Categories for the working mathematician, Second edition, Grad. Texts in Math. 5, Springer-Verlag, New York, 1998.

[MVW06] Mazza, C., Voevodsky, V., Weibel, C., Lectures on motivic cohomology, Clay Math. Monogr. 2, American Mathematical Society, Providence, RI, 2006.

[Mil80] Milne, J.S., Étale cohomology, Princeton Math. Ser. 33, Princeton University Press, Princeton, New Jersey, 1980.

[Mum68] Mumford, D., Rational equivalence of 0-cycles on surfaces. J. Math. Kyoto Univ. 9 (1968), 195-204.

[Mur85] Murre, J.P., Application of algebraic $K$-theory to the theory of algebraic cycles, in: Proc. Algebraic Geometry Sitges (Barcelona) 1983, Lecture Notes in Math. 1124, Springer-Verlag, Berlin 1985, 216-261. 
[RS16] Rosenschon, A., Srinivas, V., Étale motivic cohomology and algebraic cycles. J. Inst. Math. Jussieu 15 (2016), no. 3, 511-537.

[Sam58] Samuel, P., Relations d'equivalence en géométrie algébrique. Proc. Internat. Congress Math. 1958, 470-487.

Tohru Kohrita tohru.kohrita@fu-berlin.de

Freie Universität Berlin, Arnimallee 3, 14195 Berlin, Germany 